\title{
Impact of pore structure and fractal characteristics on the sealing capacity of Ordovician carbonate cap rock in the Tarim Basin, China
}

\author{
Jun $\mathrm{Wu}^{\text {a }, \mathrm{b}, \mathrm{c}, \mathrm{d}}$, Tailiang Fan a, b, c*, Enrique Gomez-Rivas ${ }^{\mathrm{d}, \mathrm{e}}$, Zhiqian Gao ${ }^{\mathrm{a}, \mathrm{b}, \mathrm{c}}$, Shuqing Yao ${ }^{\mathrm{e}}$, \\ Wenhuan Li ${ }^{\text {a }}$, Chenjia Zhang ${ }^{\text {a }}$, Qingqing Sun ${ }^{\mathrm{a}}$, Yu Gu ${ }^{\mathrm{a}}$, Man Xiang ${ }^{\text {a }}$ \\ a School of Energy Resources, China University of Geosciences (Beijing), Beijing 100083, China, \\ b Key Laboratory of Marine Reservoir Evolution and Hydrocarbon Enrichment Mechanism, Beijing 100083, China; \\ c Key Laboratory of Strategy Evaluation for Shale Gas, Ministry of Land and Resource, Beijing 100083, China; \\ $d$ Department of Mineralogy, Petrology and Applied Geology, Faculty of Earth Sciences, University of Barcelona, \\ Barcelona 08028, Spain; \\ e School of Geosciences, King's College, University of Aberdeen, Aberdeen AB24 3UE, United Kingdom \\ *Corresponding author: jhy17519@126.com (T. Fan)
}

\begin{abstract}
Evaluating the heterogeneity and sealing capacity of carbonate cap rocks is of great significance for hydrocarbon production. However, the main controls on pore size distribution in carbonate cap rocks are not well constrained. In this contribution we analyze drill core samples of carbonate cap rocks from the Ordovician Yingshan Formation (Tarim Basin) to study their pore properties and investigate the controls of pore-throat structure and fractal dimension on the sealing capacity of the cap rock. Results from petrography and a combination of mercury injection capillary pressure and nitrogen gas adsorption tests reveal that there are four lithological cap rock types, with micrite being the most prevalent one, followed by calcarenite. Six types of pore-throat structures and fractal dimensions (from $D_{1}$ to $D_{6}$ ) are classified based on the pore size distributions, including macropore-I, macropore-II, mesopore-I, mesopore-II, transitional pore-I and transitional pore-II. The fractal dimensions show that there is a decreasing trend from $D_{1}$ to $D_{6}$ with decreasing pore-size diameter, suggesting that the macropore and mesopore types present more complex pore-throat structures than the transitional type. The total fractal dimension $\left(D_{\text {total }}\right)$ indicates that the pore throat structures are complex and heterogeneous. The sealing heights of the gas column have a positive correlation with cement content in calcarenite and a negative correlation with microfracture density. Mesopore-II and transitional pore types make a significant contribution to enhancing the breakthrough pressure. The fractal variations mainly result from macropores and mesopores, while transitional pores and micropores weaken the impact of the whole pore system on the $D_{\text {total }}$ value. The heterogeneity and anisotropy of macropores is the main control on the sealing capacity of the cap rock. The increasing of $D_{1}$ considerably contributes to the pore shape heterogeneity and enhances the capillary resistance force to hydrocarbon migration. Therefore, the fractal dimension is an effective approach for investigating the pore throat structure and sealing capacity of carbonate cap rocks.
\end{abstract}

Keywords: Carbonate cap rock; Pore structure; Fractal characteristics; Sealing capacity.

This manuscript is published in Marine and Petroleum Geology

This is an author version of the article. For the final copy-edited version, please visit: https://www.sciencedirect.com/science/article/abs/pii/S0264817219300145

DOI: $10.1016 /$ j.marpetgeo.2019.01.014 
Wu et al., 2019. Impact of pore structure and fractal characteristics on the sealing capacity of Ordovician carbonate cap rock in the Tarim Basin, China. Marine and Petroleum Geology, DOI: 10.1016/j.marpetgeo.2019.01.014

\section{Introduction}

Cap rock, defined as a sufficiently low porous and permeable seal rock, plays a key role in hydrocarbon accumulation and preservation (e.g., Schlömer and Krooss, 1997; Corrado et al., 2014; Huq et al., 2017). Cap rock constitutes an effective barrier for the volumetric flow of hydrocarbons into overlying layers. However, cap rock should not always be regarded as a totally impermeable unit that does not allow flow of hydrocarbons or other fluids ( $\mathrm{Li}$ et al., 2005; Schmitt et al., 2013), because it may still contain relatively fine pore structures and narrow throats that allow some fluid circulation (Vavra et al., 1992; Rezaeyan et al., 2015). There are extensive studies of cap rocks composed of mudstone, shale and gypsum (Caillet, 1993; Armitage et al., 2010; Boulin et al., 2013; Corrado et al., 2014; Huq et al., 2017; Newell et al., 2017; Liu et al., 2017), but investigations on carbonate cap rocks are less abundant and have only started in recent years. Most of the few existing studies mainly focus on the identification and quantitative characterization of the carbonate cap rock (Lü et al., 2017; Wu et al., 2018a). In carbonate reservoirs, the rock heterogeneity and pore network geometry control the sealing capacity of the rock. There are recent studies of rock pore structures that determine the pore size distribution (PSD) in sandstone and shale reservoirs. However, less attention has been paid to the quantification of such distributions in carbonates because of their highly complex small-scale pore geometry characteristics (Wu et al., 2018b). Therefore, the mechanisms controlling the sealing capacity of carbonate cap rocks are not yet well understood, and systematic studies tackling this issue are required.

Unlike the millimeter and centimeter-scale pores in conventional carbonate and sandstone reservoirs (Mazzullo and Harris, 1992; Ehrenberg and Nadeau, 2005), carbonate cap rock typically presents more complicated and smaller pore structures, ranging from nanometers to several hundred microns (Wu et al., 2018b). Therefore, a complete characterization of PSD in such rock type cannot be obtained with just a single test method (Schmitt et al., 2013), making it essential to adopt a combination of techniques to properly characterize the permeability and sealing capacity of carbonate cap rocks.

There are a number of studies that have investigated the pore throat types, shapes and PSD of different carbonates using polarizing light microscope, field emission/scanning electron microscopy (FE-SEM/SEM), focused ion beam scanning electron microscopy (FIB-SEM), and micro-/nano computed tomography (Micro-CT/Nano-CT) (Anselmetti et al., 1998; He et al., 2016; Rashid et al., 2017). Among these methods, thin section petrography allows characterizing pore types and shapes at the micronscale, bur fails to achieve quantitative estimates in cases with relatively low-image resolution. For instance, features smaller than that of the maximum resolution of micrographs cannot be detected (Pant et al., 2015). Micro-CT and Nano-CT scans are 
Wu et al., 2019. Impact of pore structure and fractal characteristics on the sealing capacity of Ordovician carbonate cap rock in the Tarim Basin, China. Marine and Petroleum Geology, DOI: 10.1016/j.marpetgeo.2019.01.014

also typically utilized to visualize and reconstruct pore network systems in three dimensions (Zambrano et al., 2017). These techniques feature a maximum resolution of $50 \mathrm{~nm}$ (Pant et al., 2015; Xie et al., 2015; Zhang et al., 2017). FE-SEM/SEM and FIB-SEM can be used to investigate nanopore structures with a higher image resolution but are restricted to two dimensional analyses in the first case (Gundogar et al., 2016). In summary, all these methods outlined above are applied to qualitatively characterize pore throat structures, but do not allow their quantitative analysis due to limitations of sample size and instrument resolution.

Recently, the mercury injection capillary pressure (MICP) and nitrogen gas adsorption $\left(\mathrm{N}_{2} \mathrm{GA}\right)$ methods have shown promising results for the quantitative investigation of pore structures of shale and sandstone reservoirs (Ross and Bustin, 2009; Cao et al., 2016) and their sealing rocks (Schmitt et al., 2013). MICP and $\mathrm{N}_{2} \mathrm{GA}$ methods have been extensively utilized to quantify porosity defined by pores with radii ranging from 6 to $10,000 \mathrm{~nm}$ and from $1 \mathrm{~nm}$ to $10 \mathrm{~nm}$, respectively. The variations of PSDs obtained by these two methods are significant, revealing that neither the MICP nor the $\mathrm{N}_{2}$ GA method can be utilized alone to achieve a complete analysis of PSDs in carbonate samples (Schmitt et al., 2013; Nooruddin et al., 2014). Furthermore, there is an overlapping region of pore size between $6 \mathrm{~nm}$ to $10 \mathrm{~nm}$ that can be measured with both techniques. In order to evaluate the complex and diverse pore throat structures in carbonate cap rocks, we can take $6 \mathrm{~nm}$ as a linking point to establish the connection between MICP and $\mathrm{N}_{2} \mathrm{GA}$ measurements (Cheng et al., 2006). Then we can take the total pore volume as a benchmark to normalize and calculate the PSD in the range of 1 $\mathrm{nm}$ to $10,000 \mathrm{~nm}$. Consequently, the combination of MICP and $\mathrm{N}_{2} \mathrm{GA}$ analyses, together with thin section and SEM image analysis techniques, can provide new insights into the quantification of pore structures and their geometries in carbonate cap rock (Aliakbardoust and Rahimpour-Bonab, 2013; Schmitt et al., 2013).

The fractal dimension (D) is an important parameter to describe the irregularity and roughness of natural structures (Mandelbrot et al., 1984), and has been widely applied to investigate the heterogeneity of pore structures and irregular variations of sedimentary rocks among various reservoirs all over the world (Muller and Mccauley, 1992; Schlueter et al., 1997; Dutta and Tarafdar, 2003; He et al., 2016; Li et al., 2017). The combination of the MICP and $\mathrm{N}_{2}$ GA methods has proven to be the most effective way to determine the fractal dimension of tight sand/carbonate reservoir porosity through processing the original testing data (Aliakbardoust and Rahimpour-Bonab, 2013; Cao et al., 2015; Li et al., 2017; Yang et al., 2017).

Relatively small and narrow micro- and nanoscale pore throats not only control the excessive capillary pressure, but also provide high sealing capacity that hinders the upward migration of hydrocarbons (Schmitt et al., 2013). The lack of understanding of pore structures and their fractal dimension variations is a major handicap for the 
Wu et al., 2019. Impact of pore structure and fractal characteristics on the sealing capacity of Ordovician carbonate cap rock in the Tarim Basin, China. Marine and Petroleum Geology, DOI: 10.1016/j.marpetgeo.2019.01.014

evaluation of sealing mechanisms in carbonate cap rocks. Thus, unraveling pore throat structures and their size distribution, connectivity as well as their fractal characteristics, is essential for accurately determine the capacity of cap rock to control petroleum migration and sealing.

The main aims of the present study are (1) to systematically characterize the pore throat structure types using a combination of MICP and $\mathrm{N}_{2}$ GA methods, and thin section and SEM analyses of samples from the Lower-Middle Ordovician Yingshan $\left(\mathrm{O}_{1-2} \mathrm{y}\right)$ Formation from the Tahe oilfield, Tarim Basin; (2) to combine these analyses with the fractal theory to quantitatively distinguish the fractal characteristics of carbonate cap rock; and (3) to quantify the impact of micropore structures and their fractal dimension accurately on the sealing capacity in carbonate reservoirs.

\section{Geological setting}

The Tarim Basin is the largest one in northwestern China, with an area of $56 \times 10^{4}$ $\mathrm{km}^{2}$. The Tahe oilfield is located in the Tabei Uplift of this basin and covers approximately an area of $3,800 \mathrm{~km}^{2}$ (Fig. 1A) (Kang et al., 2005). This oilfield is located in the central-southern part of the Akekule arch in the Shaya uplift (Fig. 1B), which is bounded by the Halahatang depression to the west, the Shuntuoguole uplift to the south, the Caohu depression to the east, the Manjiaer depression to the southeast and the Luntai fault to the north (Fig. 1B) (Tian et al., 2016). Stratigraphically, the Ordovician strata can be subdivided into six formations (from old to young): Lower Ordovician Penglaiba Fm $\left(\mathrm{O}_{1} \mathrm{p}\right)$, Lower-Middle Ordovician Yingshan Fm $\left(\mathrm{O}_{1-2} \mathrm{y}\right)$, Middle Ordovician Yijianfang Fm ( $\left.\mathrm{O}_{2} \mathrm{yj}\right)$, Upper Ordovician Qiaerbake Fm $\left(\mathrm{O}_{3} \mathrm{q}\right)$, Lianglitage Fm ( $\left.\mathrm{O}_{3} \mathrm{l}\right)$ and Sangtamu Fm (O3s) (Fig. 1C) (Kang, 2005). The Yingshan Fm $\left(\mathrm{O}_{1-2} \mathrm{y}\right)$ is composed of four members $\left(1^{\text {st }}, 2^{\text {nd }}, 3^{\text {rd }}\right.$ and $4^{\text {th }}$ members $)$ from bottom to top (Wu et al., 2018a). The depositional environments of this formation gradually evolved from a restricted carbonate platform $\left(1^{\text {st }}\right.$ and $2^{\text {nd }}$ members $)$ to an open carbonate platform ( $3^{\text {rd }}$ and $4^{\text {th }}$ members) (Wu et al., 2018b).

\section{Samples and methods}

The study is based on detailed observations of $106.5 \mathrm{~m}$ of drill core from 15 wells, 105 core samples and 356 core pictures. The selected samples are all carbonate cap rocks because they can be identified using well data and drill core, fullbore microscan imaging (FMI), combination core and FMI, combination of core and wireline log, and seismic profiles (Wu et al., 2018a). These samples were studied using a series of analytical techniques, including the combination of MICP and $\mathrm{N}_{2} \mathrm{GA}$ methods, thinsection optical and electronic microscope petrography, porosity and permeability tests and fractal dimension calculations. In addition, the details of the drilling processes, well completion records and daily production data provided by the Northwest Oilfield 
Wu et al., 2019. Impact of pore structure and fractal characteristics on the sealing capacity of Ordovician carbonate cap rock in the Tarim Basin, China. Marine and Petroleum Geology, DOI: 10.1016/j.marpetgeo.2019.01.014

Branch of SINOPEC were also used in our analysis.

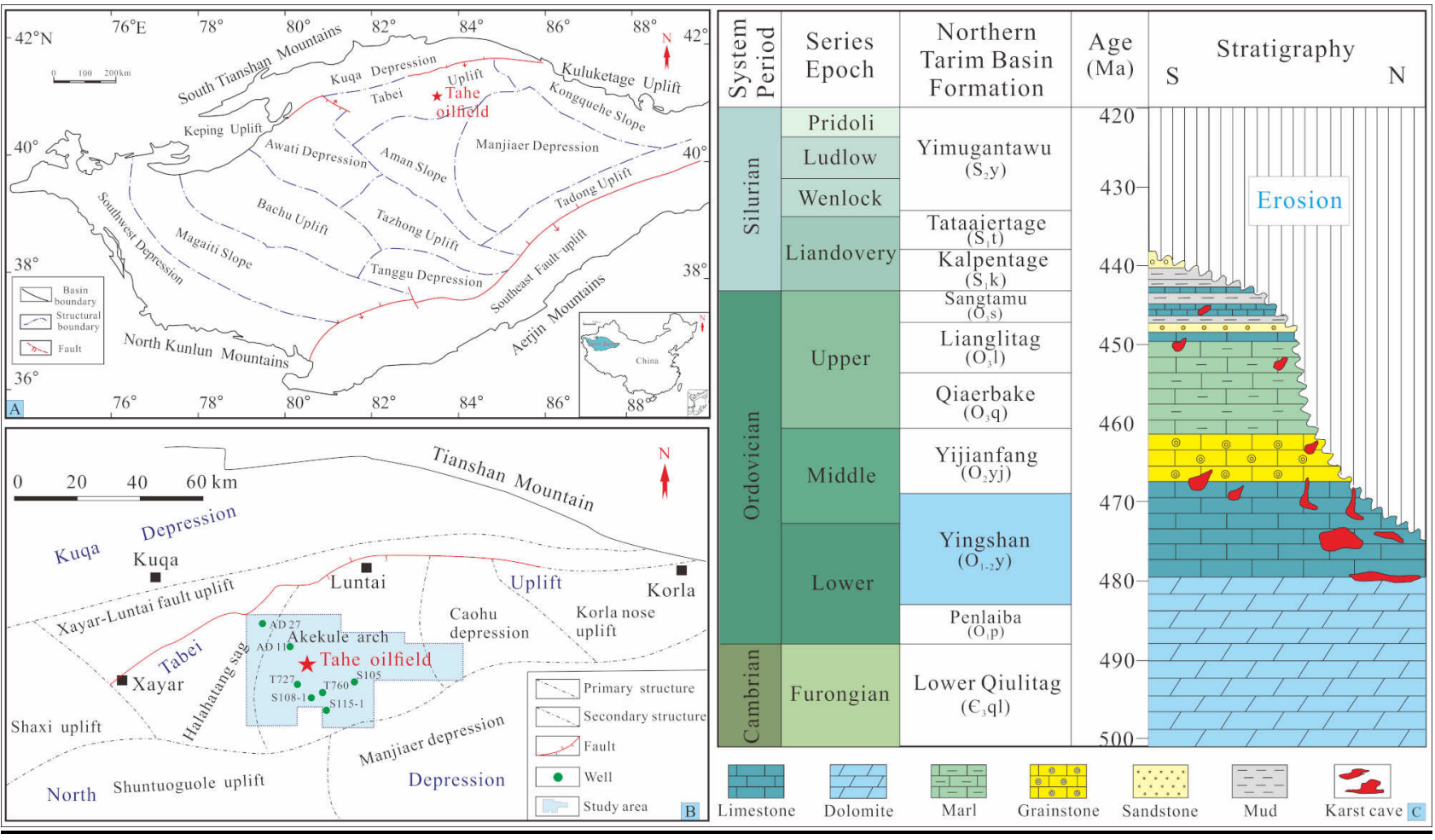

Fig. 1 (A) Geological structure map of the Tarim Basin. The Tahe oilfield is located in the Tabei Uplift; (B) Map of the studied area. The Tahe oilfield is located in the Akekule arch of the Tabei Uplift; (C) Stratigraphic column of the Tahe oilfield, from the Upper Cambrian Lower Qiulitag Fm. to the Silurian Yimugantawu Fm (modified from Wu et al., 2018a, 2018b).

\subsection{Combination of MICP and $\mathrm{N}_{2} G A$}

A total of 21 carbonate cap rock samples were analyzed from seven wells (Table 1). Each sample consisted of a cylindrical plug with a diameter of $25 \mathrm{~mm}$ and length of $50 \mathrm{~mm}$. The micropore structure data acquired from the same samples were measured using a combination of the MICP and $\mathrm{N}_{2} \mathrm{GA}$ methods at the Experimental Research Center of Wuxi Research Institute of Petroleum Geology, SINOPEC. An AUTOPORE IV9520 Micropore Structure Analyzer and JW-BK22 Surface Area Analyzer were utilized to determine the pore size distribution of the 21 samples following the Chinese standards NB/T 14008-2015, GB/T 21650.1-2008 and GB/T 21650.2-2008. We obtained a series of parameters for the quantitative evaluation of pore throat structure, including porosity, permeability, breakthrough pressure $\left(P_{b}\right)$, breakthrough radius $\left(R_{b}\right)$, median pressure $\left(P_{m}\right)$, median radius $\left(R_{m}\right)$, height of gas column (HGC), cover coefficient (CC) and specific surface area (SSA).

The breakthrough pressure $\left(P_{b}\right)$ under the gas-water phase corresponds to the point where the saturation is $10 \%$ on capillary pressure curves (Fan et al., 2011). The 
breakthrough radius $\left(R_{b}\right)$ is the radius corresponding to the breakthrough pressure. The median pressure $\left(P_{m}\right)$ corresponds to the capillary pressure at 50\% accumulated saturation of the gas-water phase. The median radius $\left(R_{m}\right)$ is the pore throat radius corresponding to the median pressure $\left(P_{m}\right)$. In addition, the breakthrough pressure of the cap rock controls the height of gas trapped in the reservoirs (Cheng et al., 2006).

The critical accumulation height of hydrocarbon gas within the reservoirs is known as the height of the cap rock gas column (HGC). The capacity of sealing natural gas of the carbonate cap rock can be expressed by the cover coefficient (CC) in specific traps (Cheng et al., 2006). The specific surface area reflects the hydraulic properties of the pore network (Zambrano et al., 2017).

$$
\begin{aligned}
& H G C=\frac{P_{\mathrm{b}}}{\left(\rho_{w}-\rho_{o g}\right) g} \\
& C C=\frac{H G C}{Z} \times 100 \%
\end{aligned}
$$

where $\rho_{w}$ and $\rho_{o g}$ are the density of formation water and oil-gas, respectively, $g$ is the acceleration of gravity and $Z$ is the closure of the structure.

\subsection{Thin section petrography}

A total of 151 thin sections were studied under polarizing light microscope at the China University of Geosciences, Beijing. 36 of these sections were 1/3-stained with Alizarin Red S to distinguish calcite and dolomite, and 15 were impregnated with epoxy resin dyed with methylene blue to highlight the pore features. Through the observation of all thin sections, the content of calcite cement obtained from at least eight photomicrographs from each thin section was determined by volumetric proportion using the point counting method via image analysis software (Wang et al., 2015).

\subsection{Porosity and permeability tests}

Effective porosity and permeability were measured on 21 plug samples (with a diameter of $25 \mathrm{~mm}$ ) at the experimental research center of Wuxi research institute of petroleum geology of SINOPEC. These tests were performed with a QK-98 Helium Gas Porosimeter and a GDS-90F Helium Gas Permeameter following Chinese standard industry methods (GB/T 29172-2012). In order to make a direct comparison between petrography and porosity-permeability properties, 21 thin sections were cut from the end of these corresponding plug samples (Erwin et al., 1979).

\subsection{Scanning electron microscopy}

Scanning electron microscopy (SEM) analyses were performed to characterize the 
pore throat geometry, internal pore structure and cement morphology in the carbonate cap rock intervals. Eight samples coated with gold were examined using a FEI Quanta FEG450 SEM with a working current set at an accelerating voltage of $20.0 \mathrm{kV}$ at the Experimental Research Center of Unconventional Technology Research institute of CNOOC.

\subsection{Fractal dimension calculation}

The number of pores with a radius larger than $r$ is counted $N(>r)$. According to the theory of fractal geometry (Mandelbrot et al., 1984), if the pore size distribution is in accordance with the fractal structure, the relationship between $N$ and $r$ can be written as (Li et al., 2017):

$$
N(>r)=\int_{r}^{r_{\max }} f(r) d r=a r^{-D}
$$

where $r_{\max }$ is the maximum pore radius, $f(r)$ is the distribution density of pore radii, $a$ is a proportionality coefficient, and $\mathrm{D}$ is the fractal dimension.

The distribution density of pore radii $f(r)$ can be obtained by deriving the pore radius $(r)$.

$$
f(r)=\frac{d N(>r)}{d r}=-D a r^{-D-1}
$$

The volume of pores with a radius smaller than $r$, termed $V(r)$, can be calculated through the integration of $r$.

$$
V(<r)=\int_{r_{\min }}^{r} f(r) \beta r^{3} d r=-\frac{D \beta a}{3-D}\left(r^{3-D}-r_{\min }^{3-D}\right)
$$

where $\beta$ is a constant associated with the geometric shape of the pore and $r_{\min }$ is the minimum radius of the pore.

Thus, the total pore volume $(V)$ can be obtained:

$$
V=-\frac{D \beta a}{3-D}\left(r_{\max }^{3-D}-r_{\min }^{3-D}\right)
$$

Using equations (5) and (6), the cumulative pore volume percentage $(S)$ of pores with a radius smaller than $r$ can be calculated:

$$
S=\frac{V(<r)}{V}=\frac{r^{3-D}-r_{\min }^{3-D}}{r_{\max }^{3-D}-r_{\min }^{3-D}}
$$

Since $r_{\min }<<r_{\max }, r$, the Eq. (7) can be simplified as follows:

$$
S=\left(\frac{r}{r_{\max }}\right)^{3-D}
$$

Now, according to the relation between capillary pressure $\left(P_{c}\right)$ and pore radius of the rock (Berg, 1975), and assuming that the wetting angle is not influenced by pore size, 
the $r_{\max }$ corresponds to the minimum capillary pressure $\left(P_{\min }\right)$ :

$$
\begin{gathered}
P_{c}=\frac{2 \sigma \cos \theta}{r} \\
P_{\text {min }}=\frac{2 \sigma \cos \theta}{r_{\text {max }}}
\end{gathered}
$$

where $\sigma$ is the interfacial tension and $\theta$ is the wetting angle.

Therefore, by combining Eqs. (9) and (10), the cumulative pore volume percentage (S) can be expressed as:

$$
S=\left(\frac{P_{\min }}{P_{c}}\right)^{3-D}
$$

In logarithm form equation (11) becomes:

$$
\lg S=(D-3) \lg P_{c}-(D-3) \lg P_{\min }
$$

where $S$ is the wetting phrase saturation corresponding to the capillary pressure $P_{c}$.

Consequently, the cross-plot between $\lg S$ and $\lg P_{c}$ will present a linear trend when the pore-throat features match the fractal theory. The slope of the straight line $(B)$ in the plot can be obtained to calculate fractal dimension as follows:

$$
D=3+B
$$

The value of $D$ in carbonate cap rocks ranges from 2 to 3 (Thompson et al. 1987), and the closer the $D$ value is to 2 , the more regular the pore throat shape and smoother the pore throat surface, whereas values of $D$ closer to 3 account for a more complex pore throat structure and rougher pore throat surface (Krohn, 1988).

It is usually straightforward to carry out a regression fitting of experimentallyderived capillary pressure data, either using the whole data set or part of it. Obviously, the values of fractal dimension obtained with this method are quite different from the actual results, and cannot truly reflect the pore-throat distribution of carbonate cap rock (Li et al., 2017). Therefore, when calculating the fractal dimension, we carried out a piece regression according to the distribution of data points and the range of pore sizes (Zhang et al., 2018). In this way we not only ensure the data integrity, but also truly reflect the pore distribution.

Based on the fractal theory, the regression of the $\lg \left(1-\mathrm{S}_{\mathrm{Hg}}\right)$ vs. $\lg \mathrm{P}_{\mathrm{c}}$ results in a completely straight line when the structures from large to small pore throats are similar, which means that the fractal dimensions are very close and can be classified as an integral fractal (single fractal). In other cases, the regression of $\lg \left(1-\mathrm{S}_{\mathrm{Hg}}\right)$ versus $\lg \mathrm{P}_{\mathrm{c}}$ shows a curved trend with various turning points. In such case, the regression curve can 
be divided into several segments to calculate the corresponding fractal dimension of distinct pore throats. According to the methods adopted by Li et al. (2017) and Zhang et al. (2018), the multi-fractal dimension in combination with the weighted average porosity was used to calculate the total fractal dimension $\left(\mathrm{D}_{\text {total }}\right)$ among the whole porethroats with various sizes.

$$
D_{\text {total }}=\sum_{i=1}^{n} \phi_{i} D_{i}
$$

where $\phi_{i}$ and $D_{i}$ are the porosity and fractal dimension of the corresponding pore-throat size, respectively.

\section{Results}

\subsection{Lithology}

Based on core and thin section observations of cap rock in the study area, four lithological types were identified: micrite, calcarenite, dolomitic limestone and silityfine crystalline dolomite (Table 1). Each of these types includes different lithological subtypes, which were grouped in order to facilitate the statistical analysis of lithologies. Specifically, intraclastic micrite and micrite are combined into the micrite type. Calcarenite includes sparry calcarenite and sparry calcirudite. Dolomitic limestone also accounts for intraclastic dolomitic limestone and micritic dolomitic limestone, while fine crystalline dolomite, argillaceous dolomite and silty crystalline dolomite are attributed to the silty-fine crystalline dolomite category (Fig. 2).

Micrite is the predominant lithological type of our cap rock. This lithology is dominantly composed of purely massive and homogenous micrite, with a small amount of scattered quartz (from zero to $2.3 \%$ in content) (Fig. 2E). This rock type is very tight, and microfractures are almost absent in the studied cores. Partially dissolved pores are occluded with calcite cements (Fig. 2D). Thin sections show that horizontal fractures and vertical or sub-vertical fractures are woven into mesh-like complex networks, and the pores are partly filled with residual bitumen (Fig. 2L).

The second most abundant lithotype is calcarenite. It is composed of gastropoda bioclasts and intraclasts ranging in size from 0.23 to $2.6 \mathrm{~mm}$ with an average of 0.75 mm (Fig. 2A, B, J, K). This type is characterized by highly compacted layers with abundant stylolites, as well as bladed or isopachous fibrous calcite cements of multiple generations. Extreme cementation (e.g. blocky cements, Fig. 2F) occludes intragranular pores, with a wide range of volumetric content of cement varying from $2.3 \%$ to $13.7 \%$ (avg. 8.6\%).

Dolomitic limestone is the third most abundant lithological type from the $2^{\text {nd }}$ and $3^{\text {rd }}$ members of the $\mathrm{O}_{1-2 \mathrm{y}}$ Formation. Euhedral rhombic dolomite crystals account for 
Wu et al., 2019. Impact of pore structure and fractal characteristics on the sealing capacity of Ordovician carbonate cap rock in the Tarim Basin, China. Marine and Petroleum Geology, DOI: 10.1016/j.marpetgeo.2019.01.014
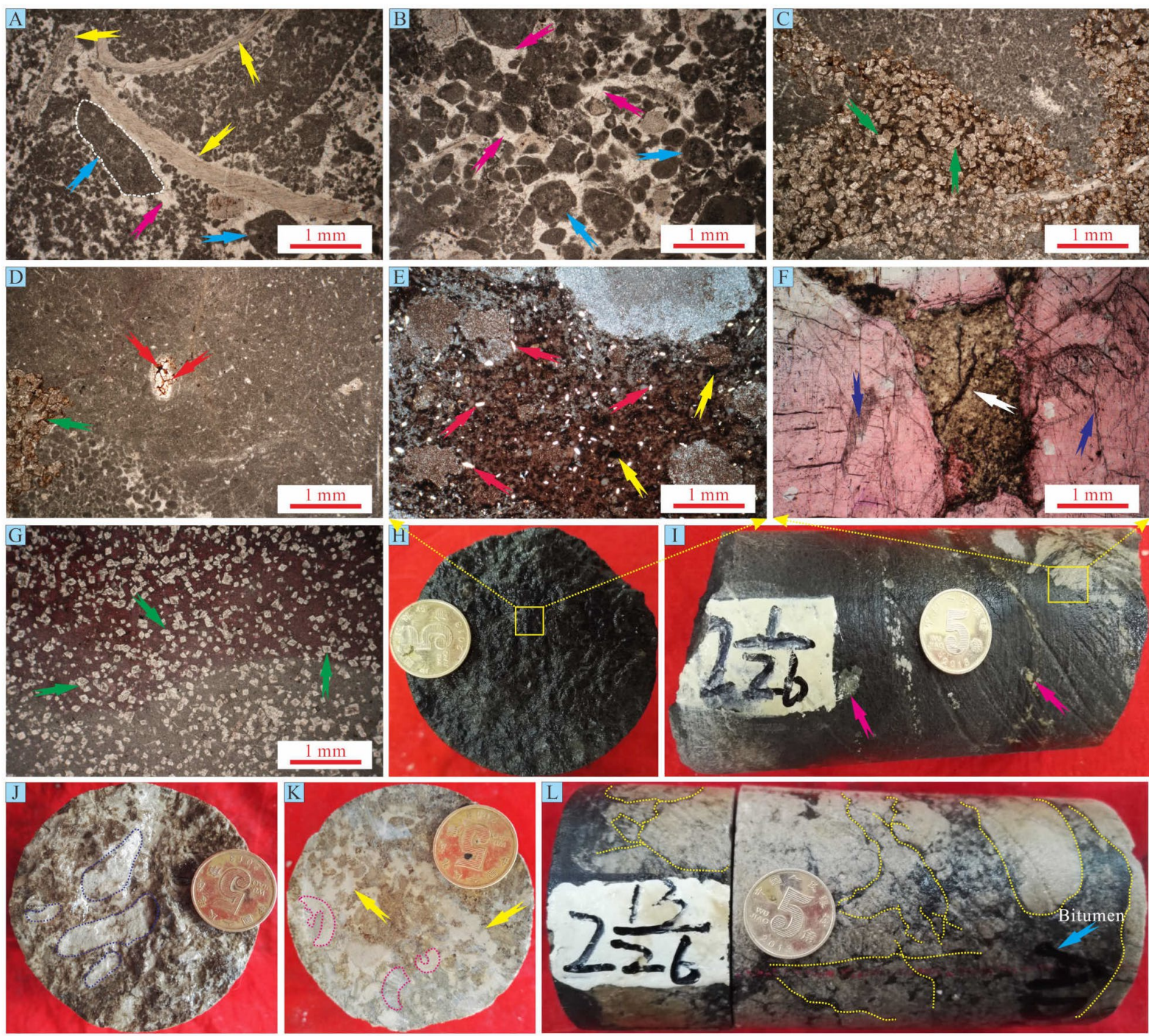

Fig. 2. Core photographs and thin section microphotographs in the dense cap rock and fracture-cavity system intervals.

(A) and (B) Calcirudite-bearing calcarenite, with locally distributed brachiopoda fragements (Strophomenid indet, yellow arrows). The individual pebbles in calcirudite (white dotted lines, blue arrows) are greater than $2.5 \mathrm{~mm}$, and the intergranular pores appear completely occluded with cements of multiple generations (pink arrows). These samples are from well T760, at a depth of $5983.69 \mathrm{~m}$. (C) Micritic dolomitic limestone, euhedral and subhedral dolomite crystals (green arrows) were highly compacted. From well T727 at $6163.66 \mathrm{~m}$ depth. (D) Dense micrite, showing the intragranular pores filled with calcite cements (red arrow). From well T727 at $6163.66 \mathrm{~m} \mathrm{depth}$. (E) and (H) Micrite contains scattered quartz (red arrows), showing oxidized black bitumen in core section or brownish oil charging traces (yellow arrows). From well AD27 at $6689.6 \mathrm{~m}$ depth. (F) and (I) Micritic dolomitic limestone, showing scattered pyrite (pink arrows), and early intercrystalline pores between calcite crystals (dark blue arrows). These pores were partly filled with dolomite crystals (white arrow). From well AD27 at $6688.92 \mathrm{~m}$ depth. (G) Micritic dolomitic limestone, euhedral rhombic dolomite crystals (blue arrows) are evenly distributed. From well AD11 at $6872.25 \mathrm{~m}$ depth. (J) Calcirudite, with intraclasts size ranging from 1.5 to $2.9 \mathrm{~mm}$ (dark blue dotted lines), without fractures and pores. From well T760 at $5978.43 \mathrm{~m}$ depth. (K) Sparry calcarenite, showing Gastropoda bioclasts (pink dotted lines). The pores resulting from dissolution appear totally filled with calcite cements (yellow arrows). From well T760 at 5975.93 m depth. (L) Micrite, horizontal and vertical fractures (yellow dotted lines) form a complex network. Pores are partly-filled with bitumen (white arrow). From well AD27 at $6691.22 \mathrm{~m}$ depth. 
Wu et al., 2019. Impact of pore structure and fractal characteristics on the sealing capacity of Ordovician carbonate cap rock in the Tarim Basin, China. Marine and Petroleum Geology, DOI: 10.1016/j.marpetgeo.2019.01.014

$30 \%-48 \%$ of the total rock volume (avg. $36.5 \%$ ) and are scattered in the host limestone (Fig. 2G). Subhedral dolomite crystals are mainly concentrated along and in the vicinity of stylolites (Fig. 2C). Residual bitumen is predominantly identified within these chemically compacted zones (Fig. 2E).

Silty-fine crystalline dolomite occupies the smallest proportion of the four lithological types and is only present in the $1^{\text {st }}$ and $2^{\text {nd }}$ members of this formation. Euhedral to subhedral rhombic silty-fine crystalline dolomite crystals are pervasive. This crystal content averages about $76.8 \%$, ranging from $57.3 \%$ to $87.6 \%$. There is almost no intercrystalline porosity in silty crystalline dolomite, and the residual pores are completely occluded with dolomite cements and pyrobitumen.

\subsection{Features of pore throat structure}

There are several published classification schemes of pore features (e.g. Xoaoth, 1966; Sing et al., 1985; Rouquerol et al., 1994; Loucks et al., 2012; Li et al., 2017). We have further subdivided the pore-throat size to analyze pore structures of carbonate cap rock based on the classification of Xoaoth (1966) and Li et al. (2017). This comprises macropore-I (with $>2500 \mathrm{~nm}$ diameter), macropore-II $(1000-2500 \mathrm{~nm})$, mesopore-I $(250-1000 \mathrm{~nm})$, mesopore-II $(100-250 \mathrm{~nm})$, transitional pore-I $(50-100 \mathrm{~nm})$, transitional pore-II $(10-50 \mathrm{~nm})$, and micropore $(<10 \mathrm{~nm})$. Features of pore throat and pore size distributions (PSDs) of carbonate cap rock were qualitatively and quantitatively analyzed through the combination of MICP and $\mathrm{N}_{2} \mathrm{GA}$ analyses (see methods section), revealing that the analyzed samples mainly comprise macropores, mesopores and transitional pores (Fig. 3, Table 1).

Other parameters were used in the analysis of the pore throat structure in this study including breakthrough pressure $\left(P_{b}\right)$, breakthrough radius $\left(R_{b}\right)$, median pressure $\left(P_{m}\right)$, median radius $\left(R_{m}\right)$, height of the cap rock gas column (HGC), cover coefficient (CC) and specific surface area (SSA). The reader is referred to the methods section for the definition of these parameters. Generally speaking, the $P_{b}$ of the carbonate cap rock varies dramatically from 0.0219 to $0.6389 \mathrm{MPa}$ (with an average of $0.1361 \mathrm{MPa}$ ). The $R_{b}$ is in the range of 223.40 to $6510.46 \mathrm{~nm}$, with a mean value of $3102.73 \mathrm{~nm}$. The $P_{m}$ ranges from 0.1717 to $5.2600 \mathrm{MPa}$, with an average of $1.2190 \mathrm{MPa}$. The corresponding $R_{m}$ varies between 27.13 and $836.17 \mathrm{~nm}$, with a mean value of $278.63 \mathrm{~nm}$. CC has a wide variation between $4.384 \%$ and $127.771 \%$, with a mean value of $27.226 \%$. The SSA is in the range of 1.8034 to $3.9148 \mathrm{~m}^{2} / \mathrm{g}$, with an average of $2.7201 \mathrm{~m}^{2} / \mathrm{g}$.

The capillary pressure $\left(P_{c}\right)$ generally increases with mercury intrusion saturation $\left(S_{H g}\right)$ in our samples. The capillary pressure curves show a very steep trend in the initial stage and have no relatively horizontal stage with gentle slopes in the intermediate stage of the mercury injection process (Fig. 3). Six types of pore throat structures in the 
Wu et al., 2019. Impact of pore structure and fractal characteristics on the sealing capacity of Ordovician carbonate cap rock in the Tarim Basin, China. Marine and Petroleum Geology, DOI: 10.1016/j.marpetgeo.2019.01.014

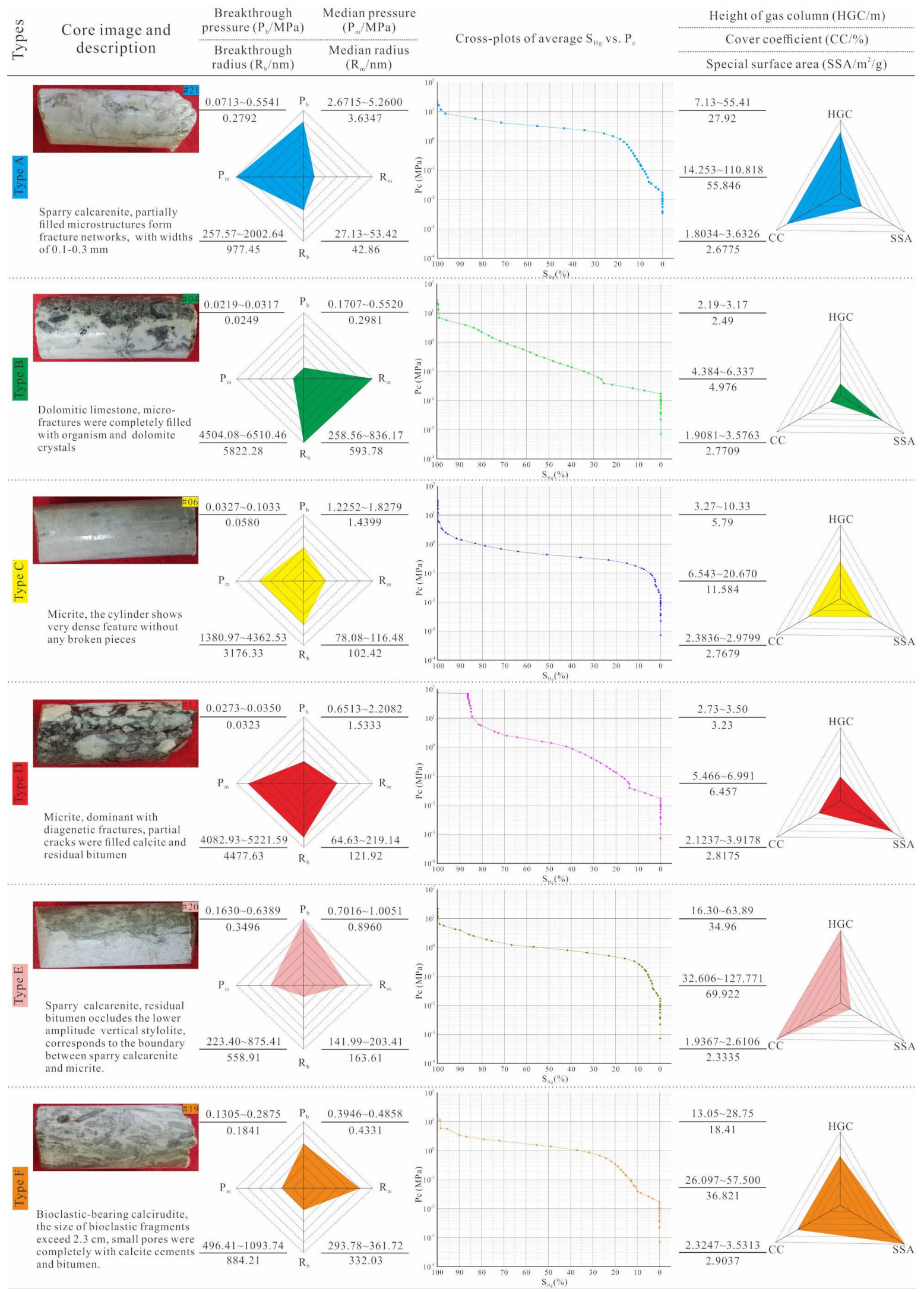

Fig. 3. Summary of the petrology, pore-throat distribution, cross-plot of pressure vs. average saturation, and sealing capacity of the six types (from type A to type F) through the combination of MICP and $\mathrm{N}_{2} \mathrm{GA}$ analyses of 21 carbonate cap rock samples, from the $\mathrm{O}_{1-2} \mathrm{y}$ Formation, Tahe oilfield. 
Wu et al., 2019. Impact of pore structure and fractal characteristics on the sealing capacity of Ordovician carbonate cap rock in the Tarim Basin, China. Marine and Petroleum Geology, DOI: 10.1016/j.marpetgeo.2019.01.014

carbonate cap rock were thus recognized based on the analysis of the aforementioned parameters, the pore throat size distribution and the cross plot of capillary pressure versus average saturation (Table 2, Fig. 3). The details are as follows:

\subsubsection{Type A}

This type features high $P_{b}$, HGC and CC, medium $R_{b}$ and low SSA (Fig. 3). The $P_{m}$ value is the highest among the six types, while the $R_{m}$ value is the lowest (Fig. 4A1).The pore throat diameter of type A shows a remarkably unimodal distribution, which lies between $25 \mathrm{~nm}$ and $250 \mathrm{~nm}$ with a mode of $34.9 \mathrm{~nm}$ (Fig. 4A2). The pore throat volumes with diameters of 25-50 nm, 50-100 nm and 100-250 nm account for $36.14 \%, 20.94 \%$ and $21.02 \%$ of the total pore volume, respectively (Fig. 4A3).

\subsubsection{Type B}

This type features the highest $R_{b}$ and $R_{m}$, medium SSA, and the lowest $P_{b}, P_{m}$, HGC and CC (Fig. 3). The $R_{b}$ and $R_{m}$ values in type B are higher than those of other five types. Conversely, the highest $R_{b}$ and $R_{m}$ values always correspond to the lowest $P_{b}$ and $P_{m}$ (Fig. 4B1), and the HGC and CC present the lowest values. For type B, the pore throat diameter shows a bimodal distribution. The larger pore throat diameter lies between 2,500 $\mathrm{nm}$ and $10,000 \mathrm{~nm}$, with a mode of $8,085 \mathrm{~nm}$, while the smaller pore throat diameter lies between $25 \mathrm{~nm}$ and $50 \mathrm{~nm}$ with a mode of $49.9 \mathrm{~nm}$ (Fig. 4B2). The volume percentage of pore-throat with diameter greater than 2,500 nm makes up $33.68 \%$ (Fig. 4B3).

\subsubsection{Type C}

This type features medium $P_{b}, P_{m}, R_{b}, \mathrm{HGC}, \mathrm{CC}$ and SSA, and lower $R_{m}$ (Fig. 3). The $R_{m}$ value of type $\mathrm{C}$ is significantly lower than that of type B (Fig. 4C1). The PSD of type $\mathrm{C}$ is dramatically unimodal with pore throat diameters mainly varying from 100 $\mathrm{nm}$ to $250 \mathrm{~nm}$ with a mode of $127.5 \mathrm{~nm}$ (Fig. 4C2). Proportionately, the pore throat with diameters in the range of $100-250 \mathrm{~nm}$ is predominant, amounting $40.02 \%$ (Fig. 4C3).

\subsubsection{Type D}

This type features high $P_{m}, R_{b}$, and SSA, medium $R_{m}$, and low $P_{b}, \mathrm{HGC}$ and CC (Fig. 3). The $P_{m}, R_{b}$, and SSA values of type D are slightly higher than those of type $\mathrm{C}$ (Fig. 5D1). However, the $P_{b}, \mathrm{HGC}$ and $\mathrm{CC}$ values of type D are much lower in comparison to those of type $\mathrm{C}$. The pore throat diameter of type $\mathrm{D}$ indicates a bimodal distribution with a larger model in the range of 2,500-1,000 nm (avg. $8085.7 \mathrm{~nm}$ ) and a smaller model in the range of 100-250 nm (avg. $127.5 \mathrm{~nm}$ ) (Fig. 5D2). The volumes of 

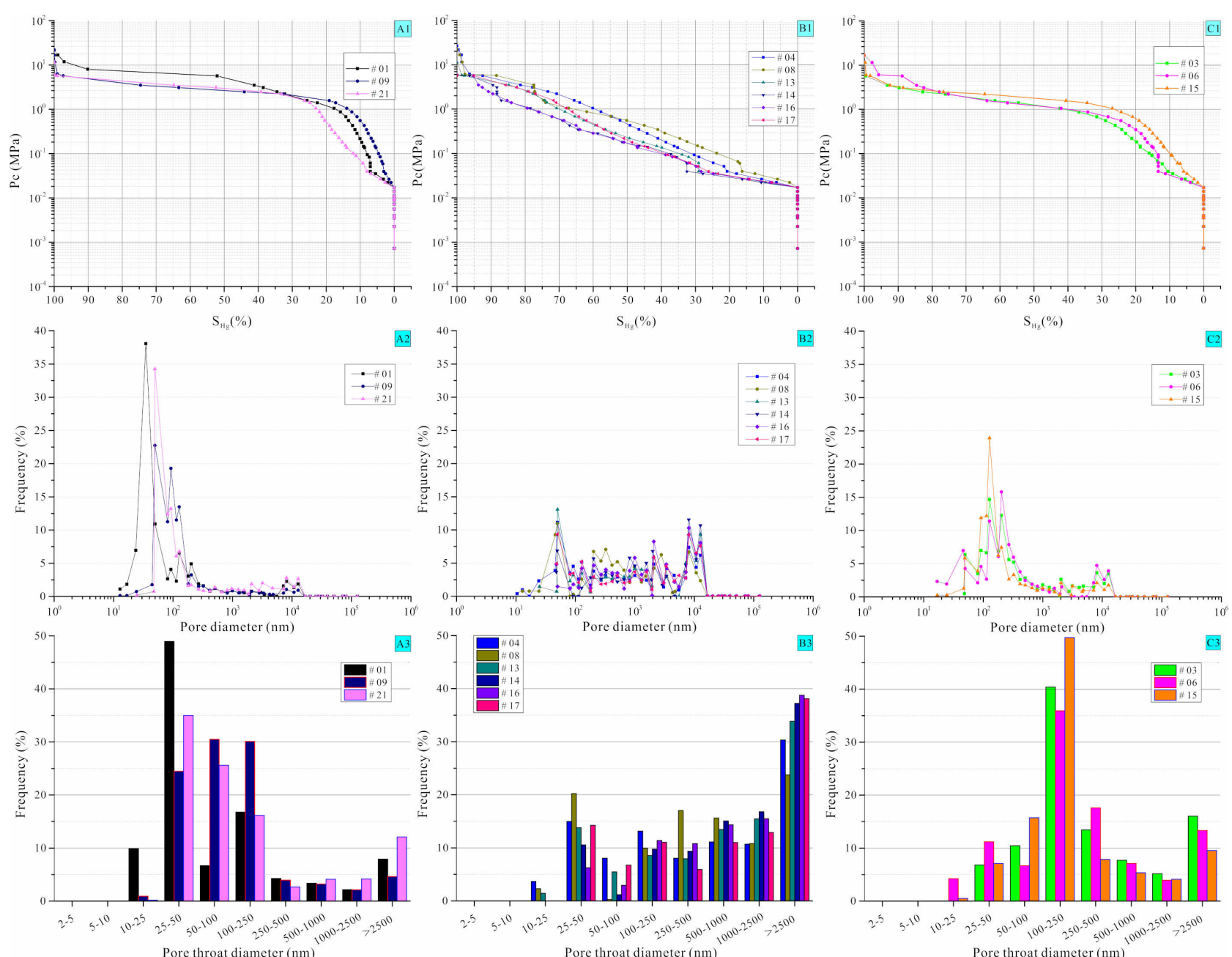

Fig. 4 Cross-plots of capillary vs. mercury saturation curves, the frequencies of pore throat sizes and their corresponding distribution intervals of types $\mathrm{A}, \mathrm{B}$ and $\mathrm{C}$ obtained from the combination of MICP and $\mathrm{N}_{2} \mathrm{GA}$ method. 

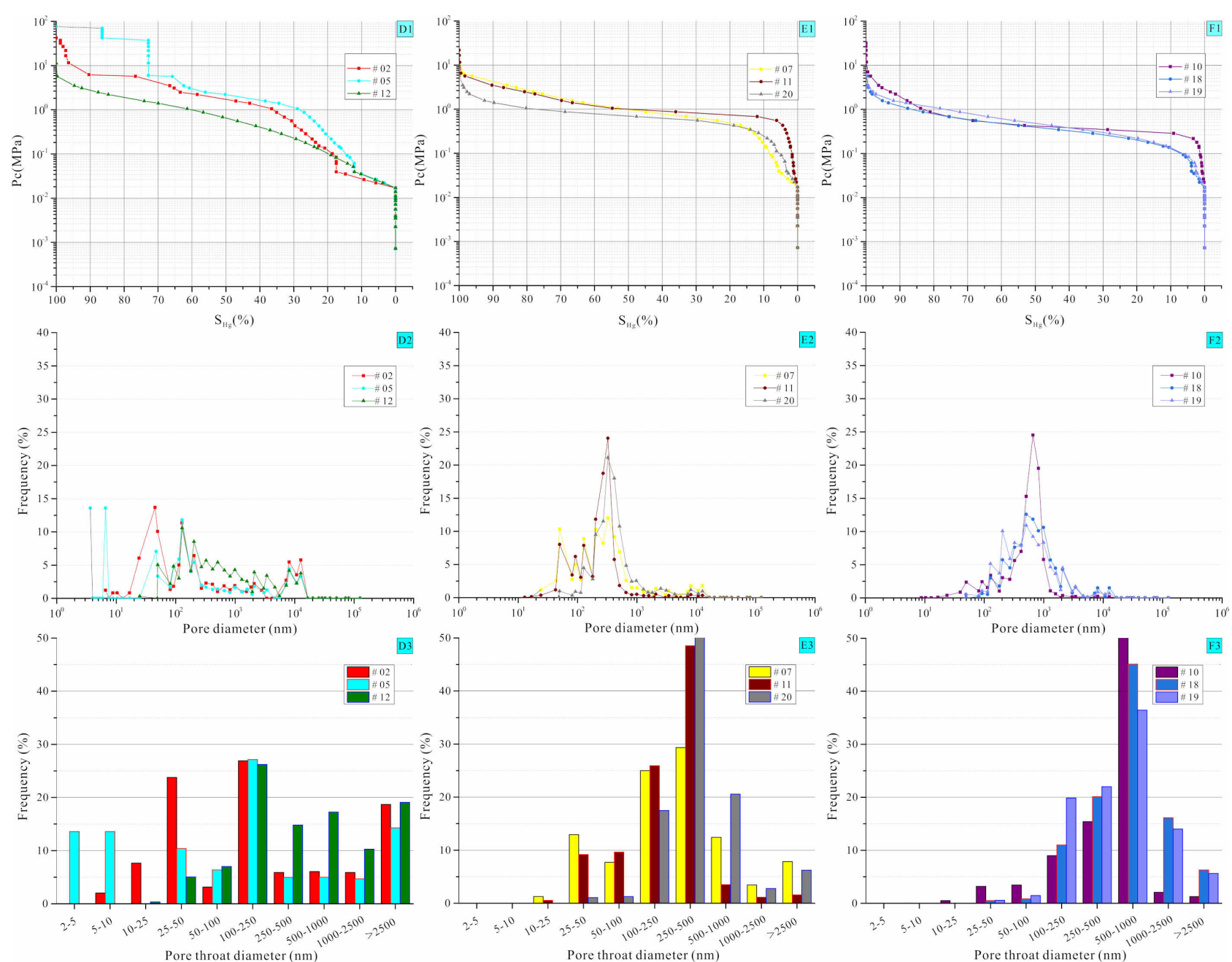

Fig. 5 Cross-plots of capillary vs. mercury saturation curves, the frequencies of pore throat sizes and their corresponding distribution intervals of types $\mathrm{D}, \mathrm{E}$, and F obtained from the combination of MICP and $\mathrm{N}_{2} \mathrm{GA}$ method. 
pore throat with a pore throat diameter of 100 to $250 \mathrm{~nm}$ and 2,500 to $10,000 \mathrm{~nm}$ account for $26.75 \%$ and $17.35 \%$ of the total respectively (Fig. 5D3).

\subsubsection{Type E}

This type features the highest $P_{b}, \mathrm{HGC}$ and $\mathrm{CC}$, medium $P_{m}$ and $R_{m}$, and the lowest $R_{b}$ and SSA (Fig. 3). The mean values of $P_{b}, \mathrm{HGC}$ and $\mathrm{CC}$ in type $\mathrm{E}$ show the largest span and are distinctly higher than those of the other types (Fig. 5E1). Compared with the other five types, the $R_{b}$ and SSA values of this type are much lower. The pore throat diameter of Type E show a considerably unimodal distribution, and the main pore throat size ranges from 250 to $500 \mathrm{~nm}$ with a mode of $323.8 \mathrm{~nm}$ (Fig. 5E2). There are $42.84 \%$ of the volume having a pore throat diameter variation of $250-500 \mathrm{~nm}$, followed by $22.80 \%$ of the total volume with a pore throat of $100-250 \mathrm{~nm}$ in diameter (Fig. 5E3).

\subsubsection{Type F}

This type features high SSA and $R_{m}$, medium $P_{b}, \mathrm{HGC}$ and CC, and low $P_{m}$ and $R_{b}$ (Fig. 3, 5F1). The SSA values are the highest among the six types of pore throat structures. For type F, there is a significant unimodal distribution of the pore-throat diameter. The pore throat diameters mainly focus on the 500 to $1,000 \mathrm{~nm}$ range, with a mode of $658.6 \mathrm{~nm}$ (Fig. 5F2). The pore volume with a pore throat diameter of 500$1,000 \mathrm{~nm}$ accounts for $48.89 \%$ of the total pore volume (Fig. 5F3).

\subsection{Fractal dimension characteristics}

By plotting $\lg \mathrm{P}_{\mathrm{c}}$ against $\lg \left(1-\mathrm{S}_{\mathrm{Hg}}\right)$ values of our carbonate cap rock samples of the $\mathrm{O}_{1-2 \mathrm{y}}$ Formation, we can relate their fractal features with different diameters of pore throats (Fig. 6, Table 2). The results indicate that not all the fractal dimensions in all carbonate cap rock samples satisfy the variation between 2.0 and 3.0 (Krohn, 1988). This is probably associated with the resolution and detection limit of the AUTOPORE IV9520 Micropore Structure Analyzer (Cheng et al., 2006; Schmitt et al., 2013). When the pore properties of carbonate rock samples are highly complex, the instrument for testing micropores inevitably cause some errors (Nooruddin et al., 2014; Zhang et al., 2018) and does not always accurately characterize the full range of pore throat sizes due to some uncertainties associated with $\mathrm{N}_{2} \mathrm{GA}$ experimental instruments (He et al., 2016). The considerable differences in the slope of the straight line for various pore throats always represent distinct fractal behaviors (Zhang et al., 2018). We further separated the cross-plots into multiple segments based on the morphology of regression curves in this plot. Six types of fractal dimensions correspond to the fractal behaviors of the macropore-I (with diameter $>2,500 \mathrm{~nm}, \mathrm{D}_{1}$ ), macropore-II $\left(1,000-2,500 \mathrm{~nm}, \mathrm{D}_{2}\right.$ ), mesopore-I (250-1,000 nm, D $)$, mesopore-II (100-250 nm, D4), transitional pore-I (50- 
$100 \mathrm{~nm}, \mathrm{D}$ ), and transitional pore-II (10-50 nm, D6) types. Then, we calculated the fractal behavior of carbonate samples using the linear fitting curves of various segments, according to the equations (12) and (13). Meanwhile, the average correlation coefficients of line regressions in multiple segments are greater than 0.965 , indicating that the carbonate cap rock samples are significantly fractal.

Consequently, the fractal dimensions following the definition range of 2.0-3.0 can be selected to reflect the fractal features of the carbonate cap rock. The results of fractal dimension for all samples are presented in Fig. 7A and Table 2. The results show that the decreasing average fractal dimension values from $\mathrm{D}_{1}$ to $\mathrm{D}_{4}$ are associated with a decrease in pore throat size. It is worth noting that the fractal dimension of the transitional pore-I type $\left(D_{5}\right)$ presents a slightly increasing trend, while the fractal dimension of the transitional pore-II type (D6) decreases (Fig. 7B).

The total fractal dimension ( $\left.\mathrm{D}_{\text {total }}\right)$ for all carbonate cap rock samples is presented in Table 2 according to the Eq. (14). Dtotal is in the range of 2.0885 to 2.9609 , with an average value of 2.6618 , indicating the pore throat structure of carbonate cap rock is complex and heterogeneous.

\subsection{Porosity and permeability}

Porosity and permeability of carbonate cap rocks from the $\mathrm{O}_{1-2} \mathrm{y}$ Formation in these six types of pore throat structures vary widely from $0.47 \%$ to $20.41 \%$ and from $0.00703 \times 10^{-3} \mu \mathrm{m}^{2}$ to $17.5 \times 10^{-3} \mu \mathrm{m}^{2}$, respectively (Table 1 ). Overall, porosity in the studied samples does not exceed 5\%, with the exception of samples \#09, \#10 and \#11 located at depths ranging from $6,688.85 \mathrm{~m}$ to $6,690.53 \mathrm{~m}$, where the average porosity increases to $12.72 \%$ (Table 1). The minimum values of average porosity and permeability are $0.91 \%$ and $0.012 \times 10^{-3} \mu^{2}$, and correspond to types B and D of pore throat structure in these carbonates, respectively. The highest values of average porosity and permeability are $9.86 \%$ and $5.860 \times 10^{-3} \mu \mathrm{m}^{2}$, and correspond to types $\mathrm{C}$ and $\mathrm{A}$ of pore throat structure in this rock (Fig. 8A). There is an extremely weak correlation between porosity and permeability, indicating that dead and/or closed pores represent a high proportion in carbonate cap rock.

\subsection{SEM analysis}

SEM images reveal that the carbonate cap rock samples are extremely dense. Microfractures (mainly $<20 \mu \mathrm{m}$ in length) and intergranular pores can be seen occasionally without appearing interconnected (Fig. 9A), resulting in different sealing potential of this rock, as discussed below. Intraclasts are mainly composed of microcrystalline calcite, with sparry calcite cement commonly partly/completely occluding the intergranular pores (Fig. 9B). 


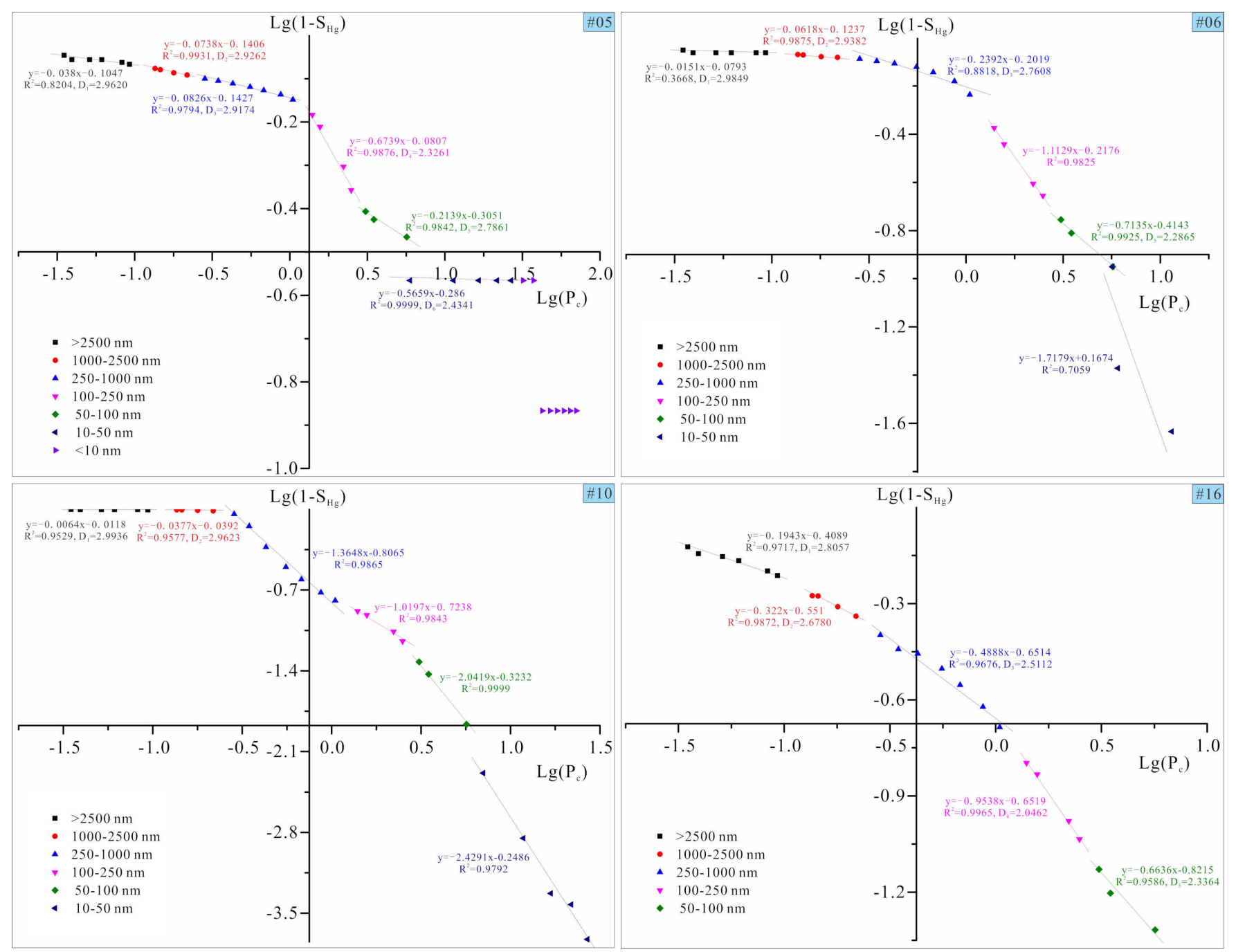

Fig. 6 Fractal dimension analysis of four selected representative carbonate cap rock samples (\#05, \#06, \#10 and \#16). 
In addition, scattered laminated illite and lamellated illite acting as pore-filling occasionally block parts of these disconnected dissolved pores (Fig. 9C, E), which yield irregularly shaped pore morphology. The amorphous intracrystalline pores and partially dissolved pores with sizes smaller than $500 \mathrm{~nm}$ are irregularly distributed in isolation (Fig. 9D, E), resulting in an enhancement of the pore network complexity. Relatively well-developed intragranular pores have complex pore shapes and are distributed among two dense micrite zones with coalescent microfabrics (Fig. 9F). Overall, the carbonate cap rock has irregular pore shapes and complex-connected pore space.
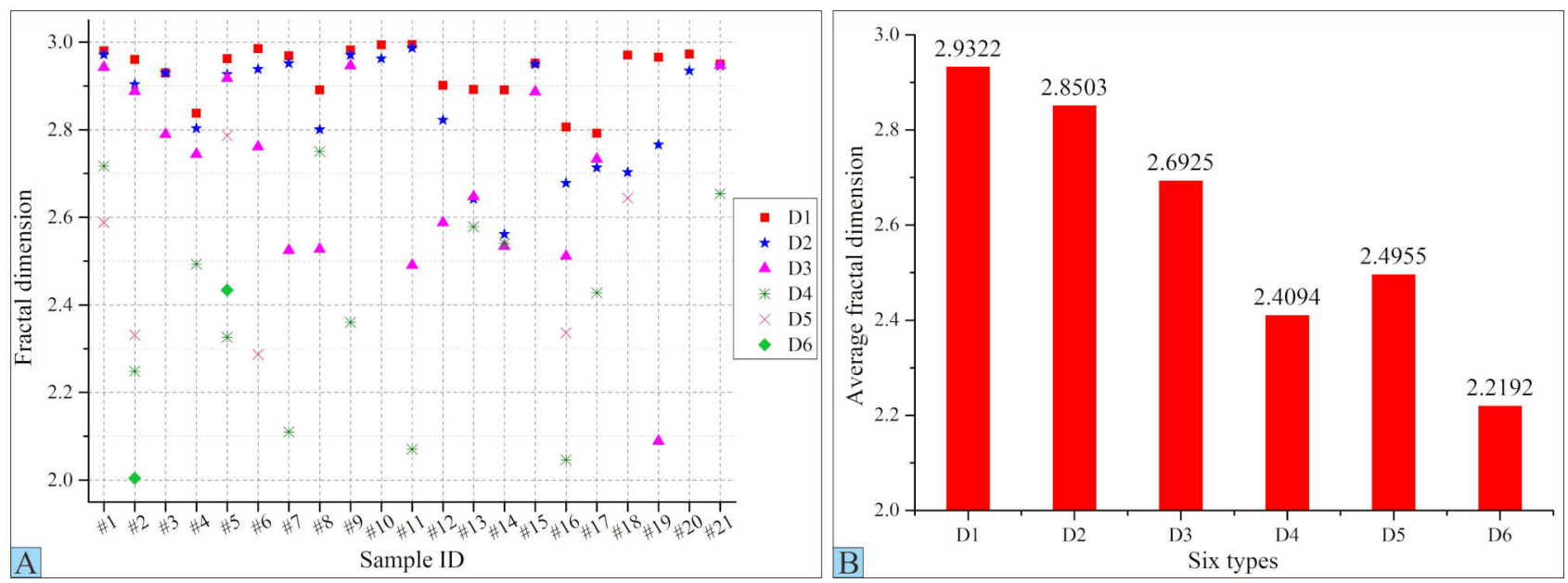

Fig. 7 (A) Six fractal dimension types from $\mathrm{D}_{1}$ to $\mathrm{D}_{6}$ that correspond to macropore-I ( $\left.>2500 \mathrm{~nm}\right)$, macropore-II (1000-2500 $\mathrm{nm})$, mesopore-I (250-1000 nm), mesopore-II (100-250 nm), transitional pore-I (50-100 nm) and transitional pore-II (10$50 \mathrm{~nm}$ ) of 21 carbonate cap rock samples in Tahe oilfield. (B) Average fractal dimensions of those six types from all samples.
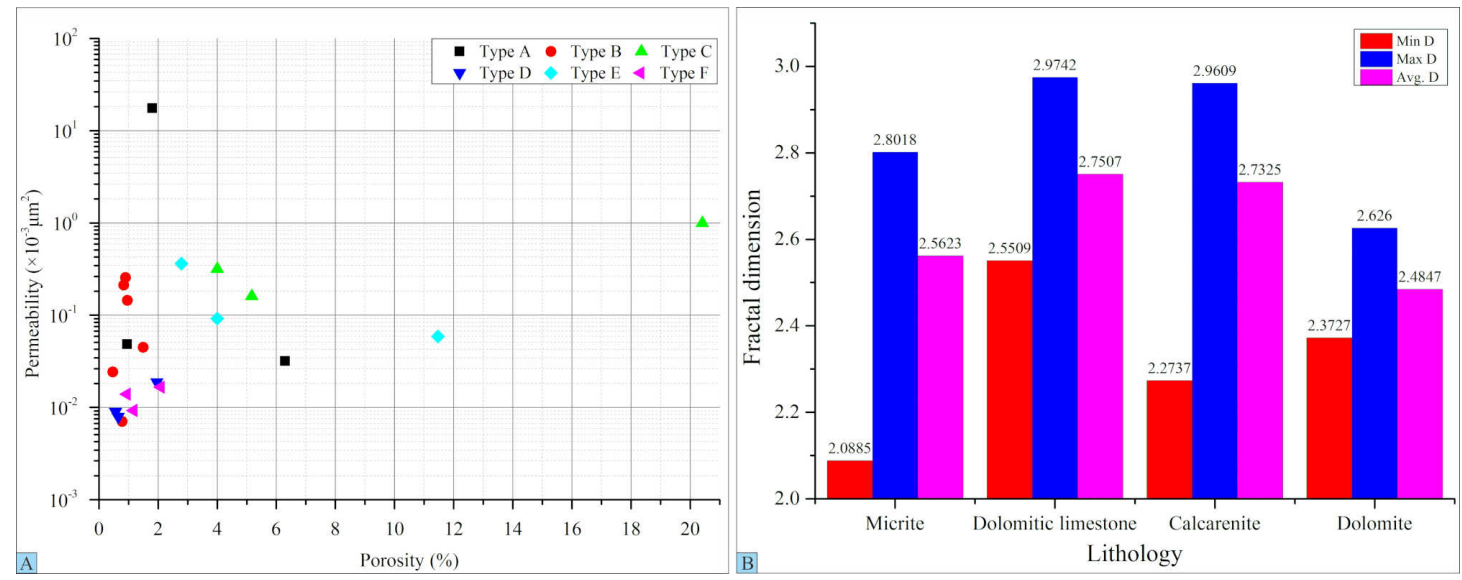

Fig. 8 (A) Cross-plot of porosity vs. permeability for six different types of pore throat structure; (B) Minimum fractal dimension, maximum fractal dimension and average fractal dimension in relation to four lithological types. 
Wu et al., 2019. Impact of pore structure and fractal characteristics on the sealing capacity of Ordovician carbonate cap rock in the Tarim Basin, China. Marine and Petroleum Geology, DOI: 10.1016/j.marpetgeo.2019.01.014
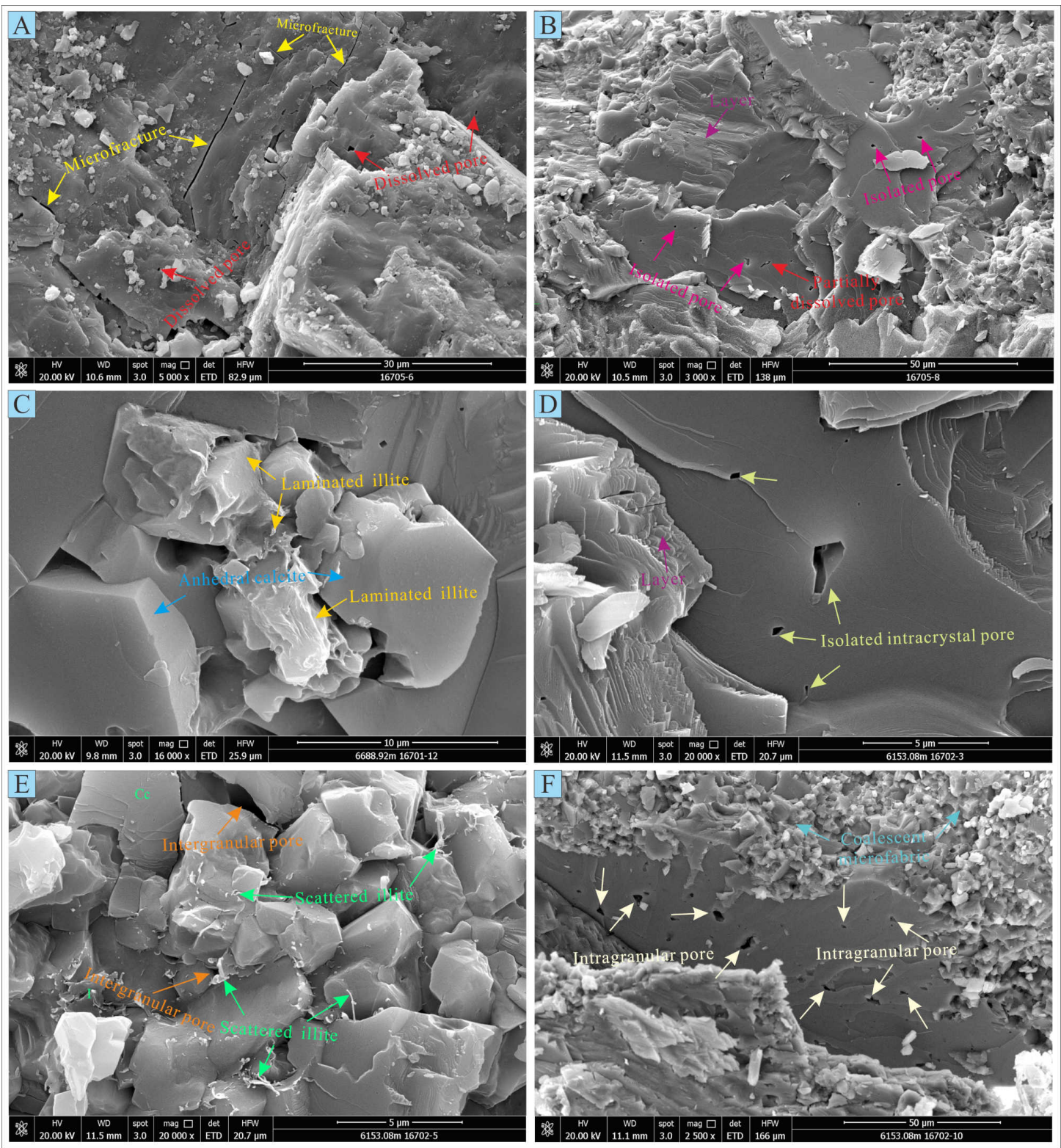

Fig. 9 Scanning electron photomicrographs of carbonate cap rock from the $\mathrm{O}_{1-2} \mathrm{y}$ Formation, Tahe oilfield. (A) Several microfractures and dissolved pores, well TS301, $6205.5 \mathrm{~m}$; (B) Isolated pores and partially dissolved pores, TS301, $6205.6 \mathrm{~m}$; (C) Laminated illite occluded the intergranular pores between anhedral calcite crystals, well AD27, $6688.92 \mathrm{~m}$; (D) Isolated intracrystal pores with various diameters, T727, $6153.08 \mathrm{~m}$; (E) Scattered illite flakes filled these intergranular pores, T727, $6153.18 \mathrm{~m}$; (F) Welldeveloped intragranular pores distributed among two dense micrite zones, and micrite crystals show coalescent microfabric, T727, $6153.21 \mathrm{~m}$. 


\section{Discussion}

\subsection{Relationship between lithology and petrophysical properties}

The porosity and permeability (poro-perm) of all samples are generally ultra-low (Fig. 10A, B), with the exception of two samples (\#10 and \#11). This is due to the presence of microfractures and dissolved pores in such samples (Fig. 10D, F). Moreover, the poro-perm values of micrite are significantly lower than those of calcarenite (Fig. $10 \mathrm{C}, \mathrm{E})$. The petrophysical properties of dolomitic limestone, particularly in vertical intervals (e.g., samples \#09 and \#10), are heterogeneous at a wide variety of scales (Table 1). Dolomite samples have much higher proportions of high-permeability values at various depths.

The cross-plot between porosity and permeability has a highly variable characteristic (Figs. 8A and 10). No positive correlation between the two parameters has been recognized in the micrite, calcarenite and silty-fine crystalline dolomite lithologic types (Fig. 10C, E, F), though a good positive correlation characterizes the dolomitic limestone lithotype with a high correlation coefficient of 0.8413 (Fig. 10D). An increase in porosity is not related to an increase in permeability, indicating that carbonate cap rock has poorly-connected pore throat systems (Jaya et al., 2005; Norbisrath et al., 2017; Rashid et al., 2017). This is certainly the case of the carbonate cap rock in the present study, considering that it is dominated by the four lithological types described above (Armitage et al., 2010; Zambrano et al., 2017). There are also wide porosity and permeability variations between the six types of pore throat structures (from type A to F) as well as in each sample within a single pore throat structure type (Table 1). This indicates that the carbonate cap rock from the $\mathrm{O}_{1-2} \mathrm{y}$ Formation in the Tahe oilfield has strong heterogeneity and significant anisotropy, both probably related to the presence of multiple pore throat structures induced by microfractures during mechanical compaction and cementation (Wu et al., 2018b).

The maximum fractal dimension and average fractal dimension measured in samples corresponding to the calcarenite and dolomitic limestone lithotypes are greater than 2.0 and closer to 3.0 (Fig. 8B). The average fractal dimensions of micrite and dolomite are significantly lower than those of the calcarenite and dolomitic limestone. The results indicate that calcarenite and dolomitic limestone present more complex pore surfaces. Our results reveal that the pore throat structures of carbonate cap rock are influenced by the lithological type of carbonates formed in the process of sedimentary rock accumulation or during diagenesis (Schmitt et al., 2013; Wu et al., 2018b). The fractal dimension variability reflects the combined and interrelated effected of mechanical and chemical compactions, dissolution and cementation. 
Wu et al., 2019. Impact of pore structure and fractal characteristics on the sealing capacity of Ordovician carbonate cap rock in the Tarim Basin, China. Marine and Petroleum Geology, DOI: 10.1016/j.marpetgeo.2019.01.014
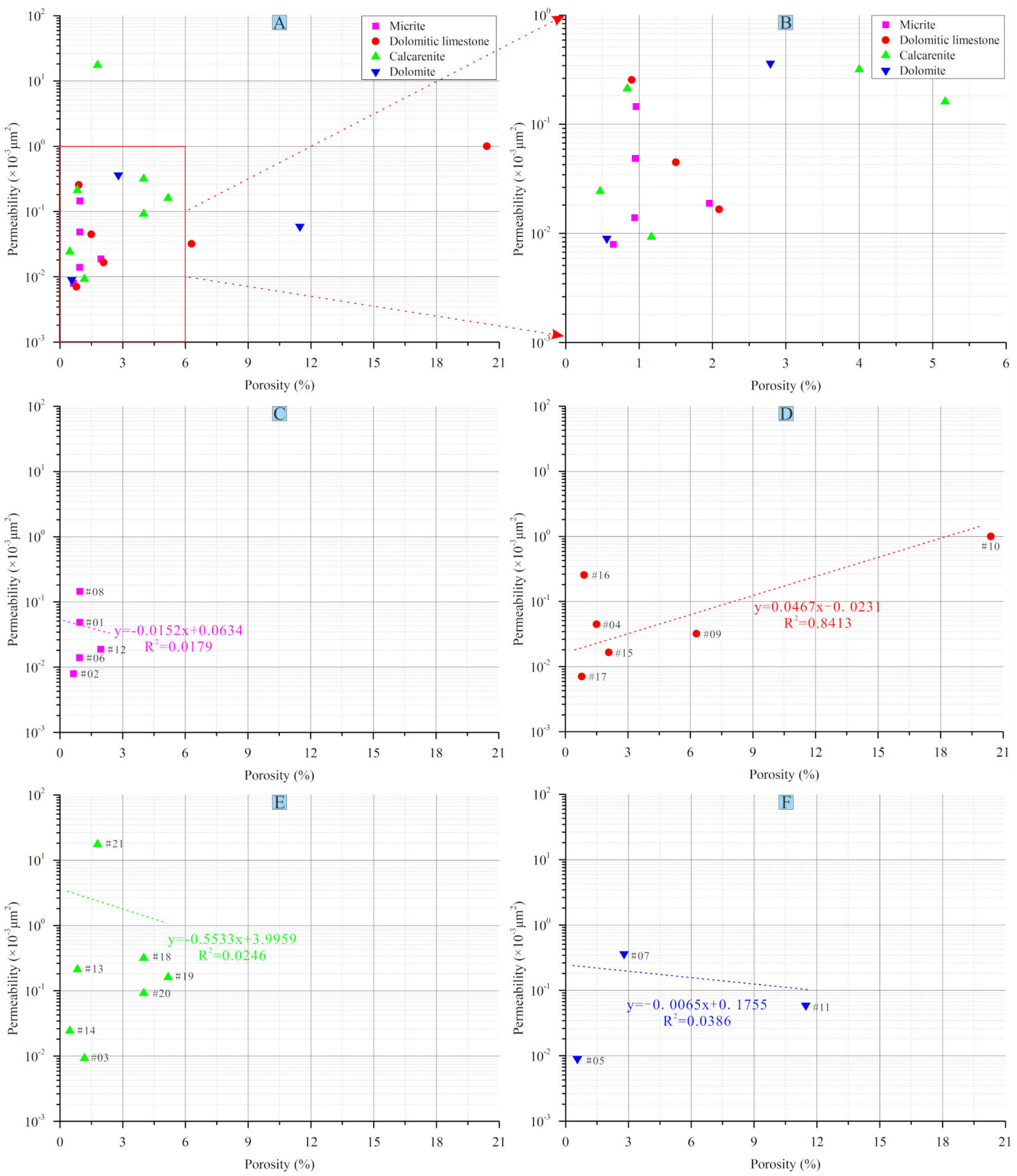

Fig. 10 (A) Cross-plot of porosity vs. permeability in relation to four lithological types for carbonate cap rock; (B) Partial enlarged graph of porosity vs. permeability for the four lithological types; (C), (D), (E) and (F) Cross-plot for micrite, dolomite limestone, calcarenite and dolomite respectively. 

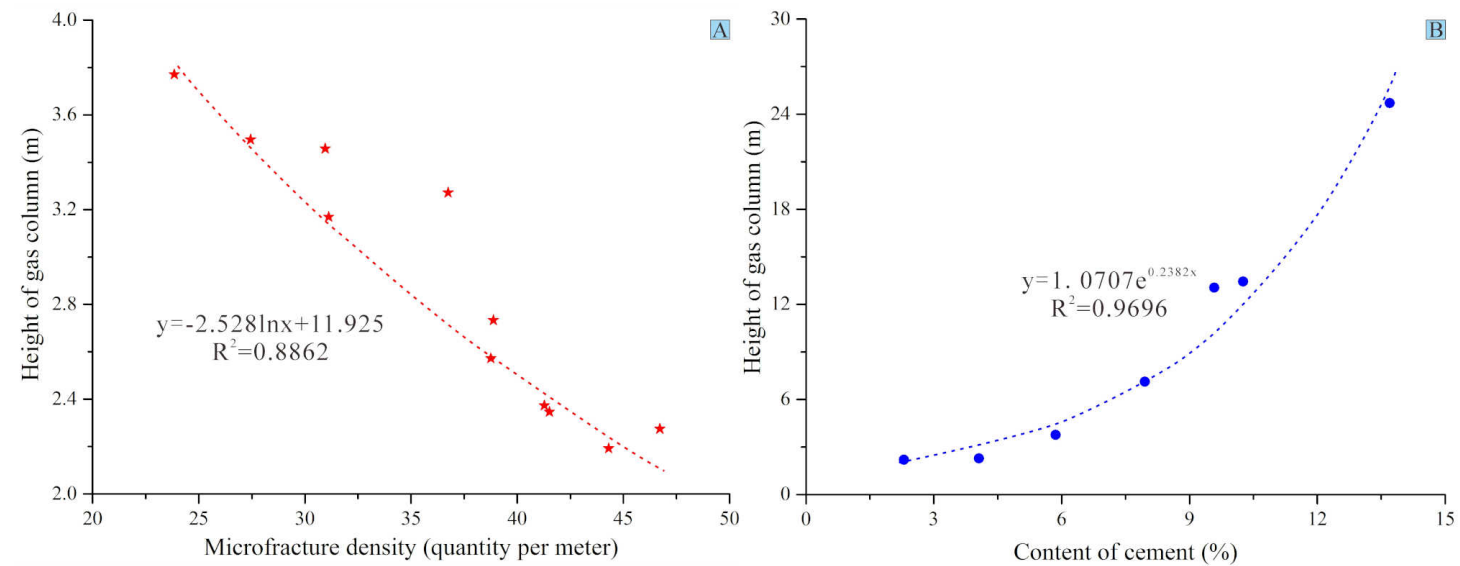

Fig. 11 (A) Microfracture density vs. height of gas column for carbonate cap rock in poor sealing types $\mathrm{B}$ and D of pore throat structure; (B) Content of cement vs. height of gas column in seven calcarenite carbonate cap rock samples (samples \#03, \#13, \#14, \#18, \#19, \#20 and \#21).

\subsection{Controls of microfractures and cementation on the cap rock sealing capacity}

The sealing capacity of a reservoir seal can be reflected by the sealing height of the gas column (HGC) (Cheng et al., 2006), in a way that the greater the value of HGC, the stronger the sealing capacity of the rock is and vice versa. The relationship between microfracture density (defined as the number of microfractures per meter), cement content and HGC have been analyzed in order to determine the controls of microfracture and cementation on the sealing capacity of the carbonate cap rock.

The HGC has a significantly good negative correlation with microfracture density in the pore throat structure types B and D (poor sealing capacity), with a higher correlation coefficient (Fig. 11A). The HGC for carbonate cap rock gets reduced with increasing microfracture density, conforming to the rule of the logarithmic function (Fig. $11 \mathrm{~A})$. This is maybe because the sealing capacity of the cap rock is strongly dependent on the disconnection of the pore throats (Schmitt et al., 2013; Rezaeyan et al., 2015; Zambrano et al., 2017). Porous microfracutures related to calcite cement make the isolated intergranular pores to be connected in sample \#21 (with a permeability of $17.5 \times 10^{-3} \mu \mathrm{m}^{2}$, Fig. 10E and Table 1), and thus provide more favorable channels for the upward migration of hydrocarbons (Fig. 9A) (Newell et al., 2017). There are approximately four or five orders of magnitude difference in the permeability of the studied samples containing microfractures versus those without them (Table 1).

Additionally, comparisons between lithology and cover coefficients for the 21 samples analyzed using the combination of MICP and $\mathrm{N}_{2} \mathrm{GA}$ methods shows that the micrite and silty-fine crystalline dolomite lithotypes with very fine micrite crystals and dolomite crystals do not always present high cover coefficient values (Table 1). This finding is inconsistent with previous studies (Schmitt et al., 2013; Pant et al., 2015). 
This phenomenon could be explained considering the presence of microfractures, resulting in a poor sealing capacity of these lithological types in relation to hydrocarbon accumulation (Zhou et al., 2017). Consequently, the occurrence of microfractures strongly facilitates the effective connectivity of pores and throats (Ehrenberg and Nadeau, 2005) and provide connected pathways to fluid flow (Ross and Bustin, 2009; Rashid et al., 2017), especially for closed pores or non-connected voids, and then possess an adverse influence on the sealing performance of the carbonate cap rock.

For the calcarenite, the HGC is positively correlated with the cement content with a high correlation coefficient. The HGC increases with cement following an exponential form (Fig. 11B), indicating that there is significant porosity occlusion during multiple cementation. This is ascribed to the chemical reactivity of calcite minerals during the cementation process (Budd, 2002). The reduction of intergranular porosity is supposed to be caused by pervasive cementation. Calcite cement occludes pore throat channels and considerably reduces their connectivity (Fig. 2F). This is one of the dominant contributors to the effective blocking of hydrocarbon migration (Rezaeyan et al., 2015). The carbonate cap rocks of the Yingshan Fm. in this studied area are of a better sealing capacity because of increasing widespread occurrence of cement content.

Dissolution of calcite minerals by aggressive fluids occurs in the carbonate cap rocks having a relative higher porosity (Mazzullo and Harris, 1992; Esteban and Taberner, 2003), and poses an effective compensation for porosity losses caused by chemical compaction and calcite cementation (Fig. 2K) (Rashid et al., 2017). However, dissolution has a limited enhancement effect on the permeability of the cap rock. This is attributed to the fact that additional porosity is generally in the form of pore voids and isolated pores rather than in the form of pore throat channels for all samples within largely unchanged untra-low porosity and permeability (Colón et al., 2004; Rashid et al., 2017).

The originally formed intergranular pores and microstructures always undergo strong chemical reactivity of carbonate minerals, affected by the strong mechanical compaction, compaction solution and multiple cementation together (Javanbakht et al., 2018). This probably results in a rapid reduction of porosity and permeability during relative deep burial (Budd, 2002; Ehrenberg and Nadeau, 2005). These microstructures and intergranular pores are partly/completely blocked by calcite cements and/or laminated illites (Fig. 9, Rashid et al., 2017), and thus they could not act as connected flow pathways for oil-gas migration individually. 
Wu et al., 2019. Impact of pore structure and fractal characteristics on the sealing capacity of Ordovician carbonate cap rock in the Tarim Basin, China. Marine and Petroleum Geology, DOI: 10.1016/j.marpetgeo.2019.01.014

\subsection{Controls of breakthrough pressure and median pressure on porosity}

The correlations between the breakthrough pressure $\left(P_{b}\right)$, median pressure $\left(P_{m}\right)$ and six porosity types (from macropore-I to transitional pore-II) were analyzed further and compared in Figs. 12, 13 and 14. The $P_{b}$ shows almost no correlation with the marcopore-I and macropore-II types (Fig. 12A1, B1), and a very weak positive correlation with mesopore-I type (Fig. 13C1). However, the $P_{b}$ has a good positive linear correlation with the mesopore-II, transitional pore-I and transitional pore-II types with correlation coefficients higher than 0.71 (Fig. 13D1, 14E1, F1). This suggests that the breakthrough pressure of the cap rock is strongly controlled by the well-developed mesopore-II, transitional pore-I and transitional pore-II types that affect the trigger threshold of hydrocarbon migration (Li et al., 2005; Fan et al., 2011).

In addition, the $P_{m}$ has an obvious negative correlation with the marcopore-I and macropore-II types, with low correlation coefficients (Fig. 12A2, B2), and a weak negative correlation with the mesopore-I type, with an extremely low correlation coefficient (Fig. 13C2). $P_{m}$ shows no correlation with the mesopore-II and transitional pore-I types (Fig. 13D2, 14E2), and there is only a slightly positive relation between $P_{m}$ and the transitional pore-II type (Fig. 14F2). A comparison of the predominant pore size distribution reveals that the volume of macropores and mecropores makes up to $47.37 \%$ and $33.93 \%$ in the type B pore structure, respectively (Fig. 4B2), while it adds up to $17.32 \%$ and $61.71 \%$ of the total pore volume in type $\mathrm{C}$ (Fig. $4 \mathrm{C} 2$ ). The total fractal dimension of type $B$ is 2.5971 , which is significantly smaller than that of type $C(2.8500)$ (Table 2). These results imply that, with the decreasing proportion of macropores and increasing proportions of mesopores in the total volume, the pore networks of carbonate cap rock are more heterogeneous and prevent hydrocarbon flow because of their tightness (Huq et al., 2017; Lü et al., 2017). Accordingly, the sealing capacity of carbonate cap rocks becomes higher when the relative presence of mesopores with respect to macropores is higher. This can be explained by the significant role of the relatively small pore throat radii of cap rock in hindering the petroleum fluid transport through the overlying seal rocks (Schlömer and Krooss, 1997; Boulin et al., 2013).

\subsection{Relationship between fractal dimension and pore structure parameters}

A range of fractal dimensions of pore throats can be obtained from the combination of MICP and $\mathrm{N}_{2} \mathrm{GA}$ analyses (Table 2). With increasing fractal dimension, carbonate cap rocks become more heterogeneous (Xie et al., 2015). $\mathrm{D}_{1}$ and $\mathrm{D}_{2}$ are larger than $\mathrm{D}_{3}$, D4, D5 and $\mathrm{D}_{6}$ (Fig. 7B), thus indirectly indicating that the homogeneity of systems defined by mesopore and transitional pore type structures are better than those with a macropore type structure. The macropore types have more complex pore networks. Average $\mathrm{D}_{1}$ and $\mathrm{D}_{2}$ values are far from 2.0 and closer to 3.0 (Fig. 7B), meaning that the macropore structures with a discrete irregular PSD are heterogeneous with a highly 
Wu et al., 2019. Impact of pore structure and fractal characteristics on the sealing capacity of Ordovician carbonate cap rock in the Tarim Basin, China. Marine and Petroleum Geology, DOI: 10.1016/j.marpetgeo.2019.01.014

complex pore system. Additionally, average $\mathrm{D}_{5}$ and $\mathrm{D}_{6}$ approach a value of 2.0, indicating that the transitional pore structures are more homogeneous (Thompson et al. 1987).

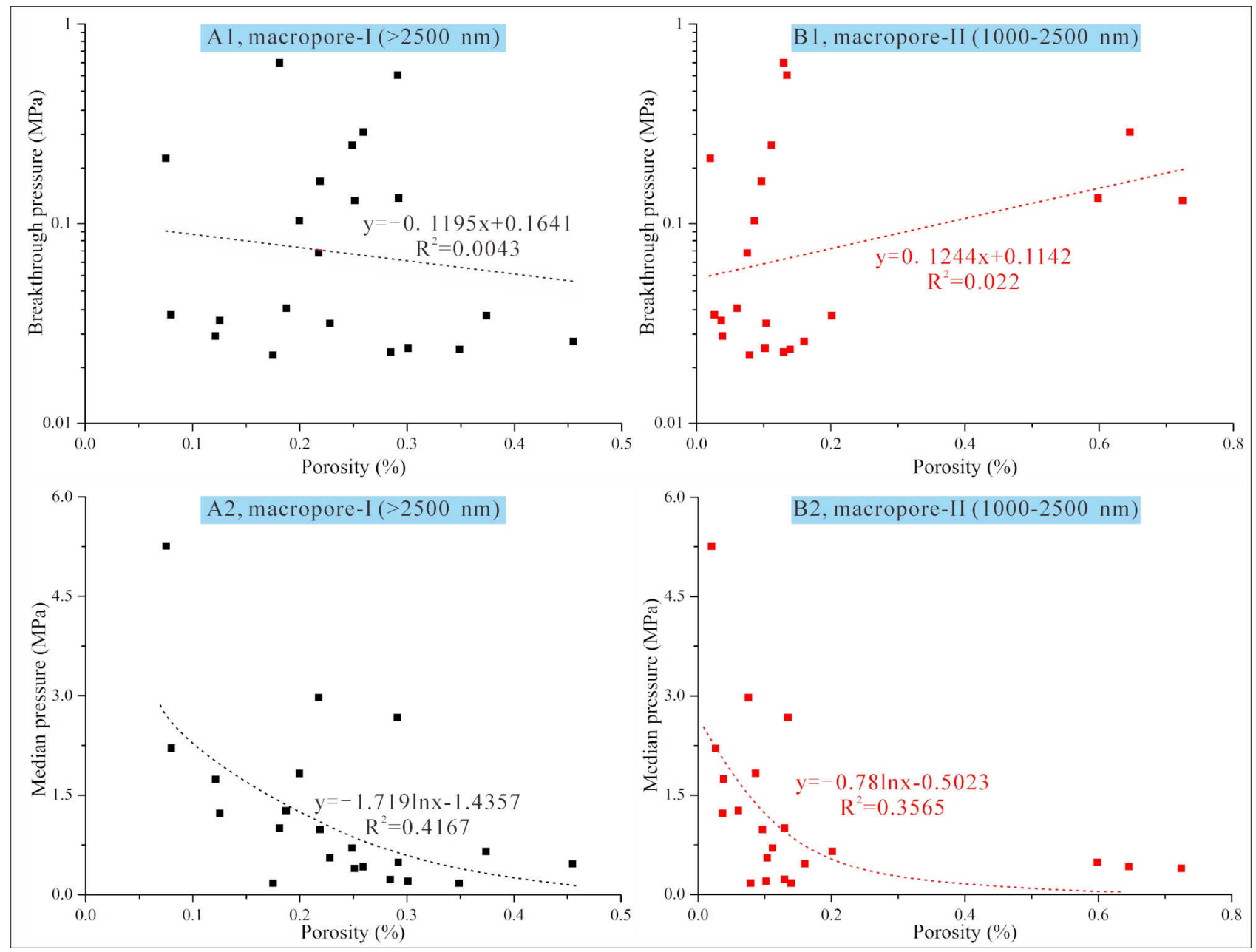

Fig. 12 Relations between porosity (macropore-I and macropore-II) and the breakthrough pressure and median pressure from the combination method of the MICP and the $\mathrm{N}_{2} \mathrm{GA}$ for 21 carbonate cap rock samples. 
Wu et al., 2019. Impact of pore structure and fractal characteristics on the sealing capacity of Ordovician carbonate cap rock in the Tarim Basin, China. Marine and Petroleum Geology, DOI: 10.1016/j.marpetgeo.2019.01.014

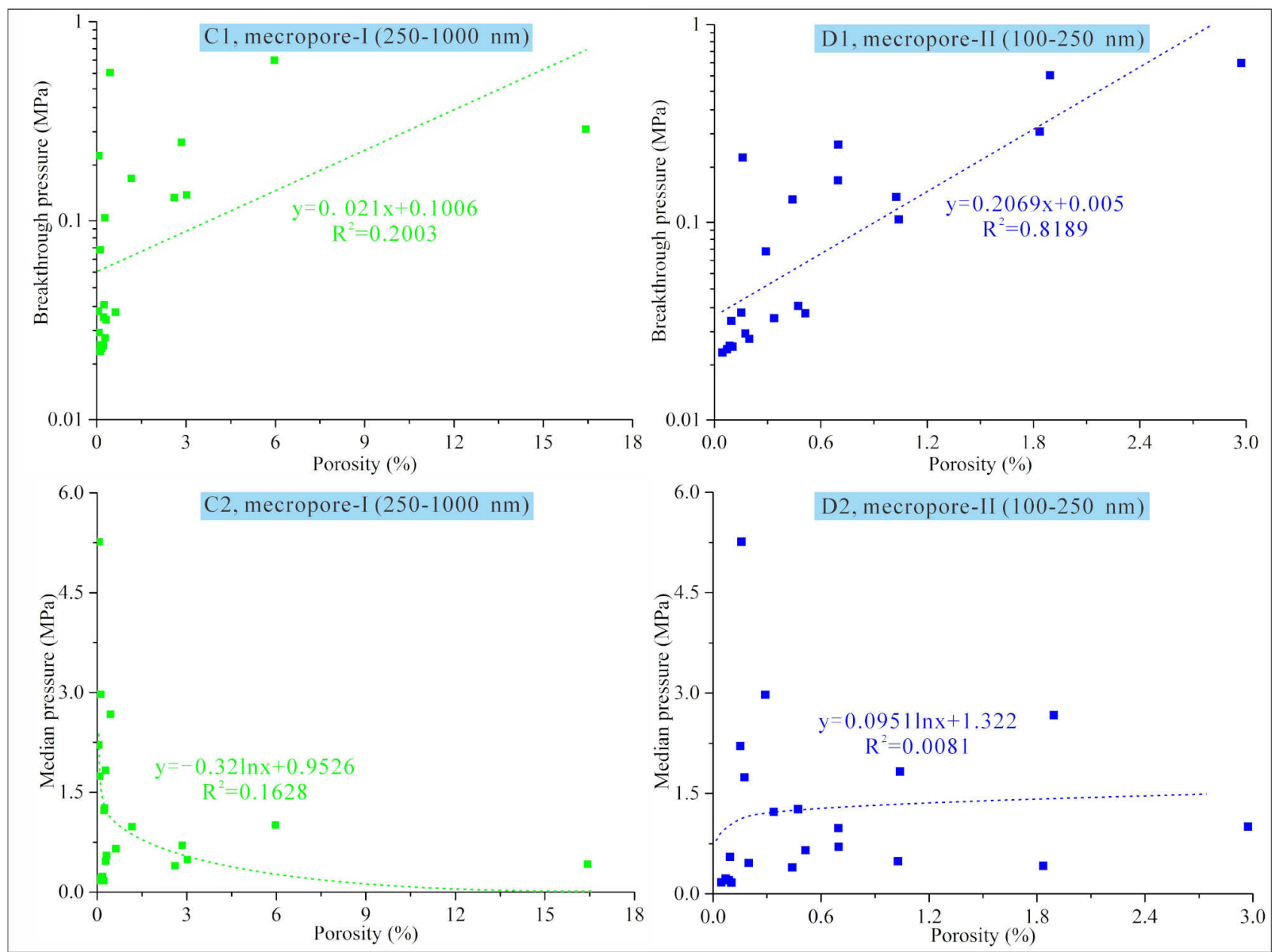

Fig. 13 Relations between porosity (mecropore-I and mecropore-II) and the breakthrough pressure and median pressure from the combination of MICP and $\mathrm{N}_{2} \mathrm{GA}$ methods for 21 carbonate cap rock samples. 
Wu et al., 2019. Impact of pore structure and fractal characteristics on the sealing capacity of Ordovician carbonate cap rock in the Tarim Basin, China. Marine and Petroleum Geology, DOI: 10.1016/j.marpetgeo.2019.01.014
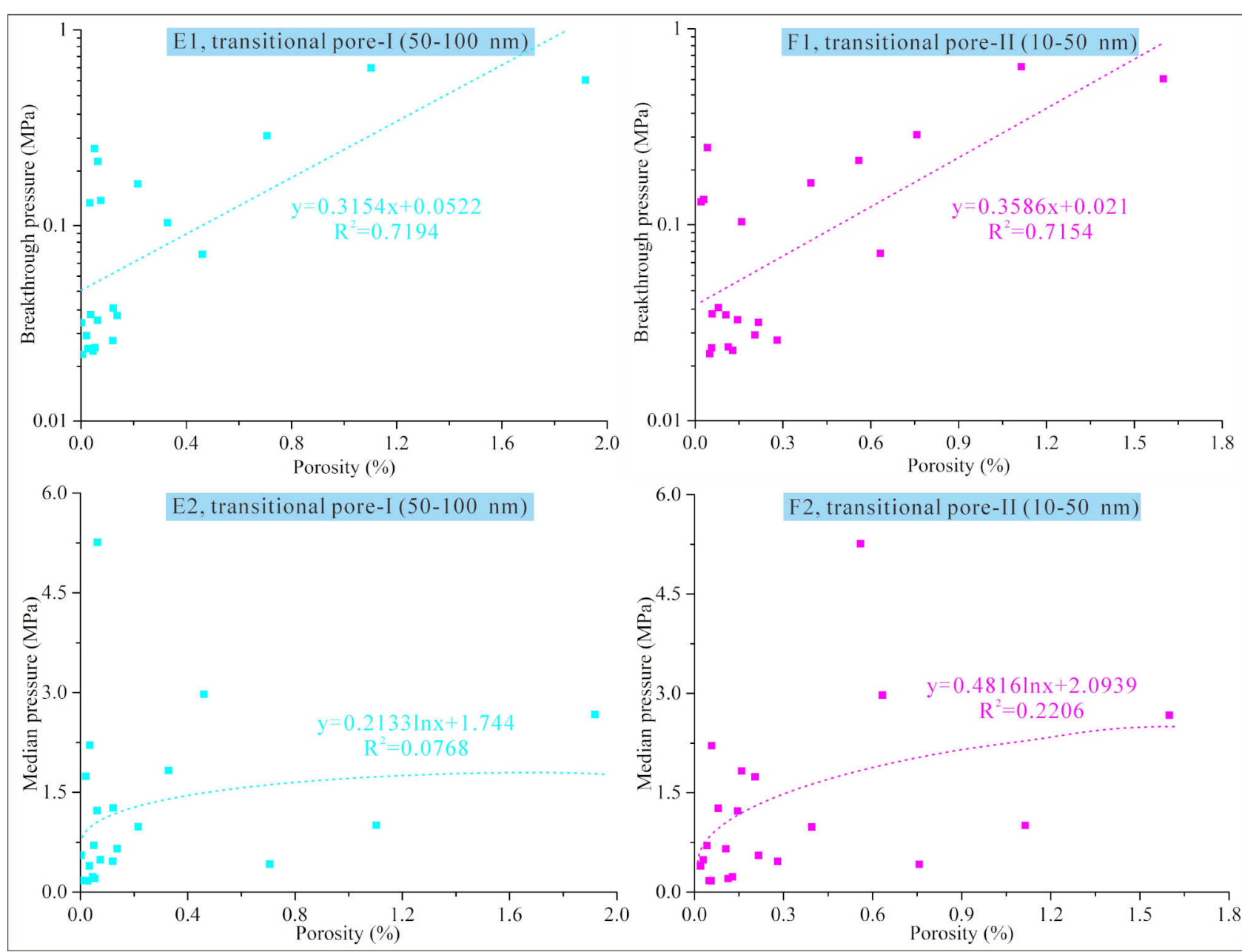

Fig. 14 Relations between porosity (transitional-I and transitional-II) and the breakthrough pressure and median pressure from the combination of the MICP and the $\mathrm{N}_{2} \mathrm{GA}$ methods for 21 carbonate cap rock samples. 

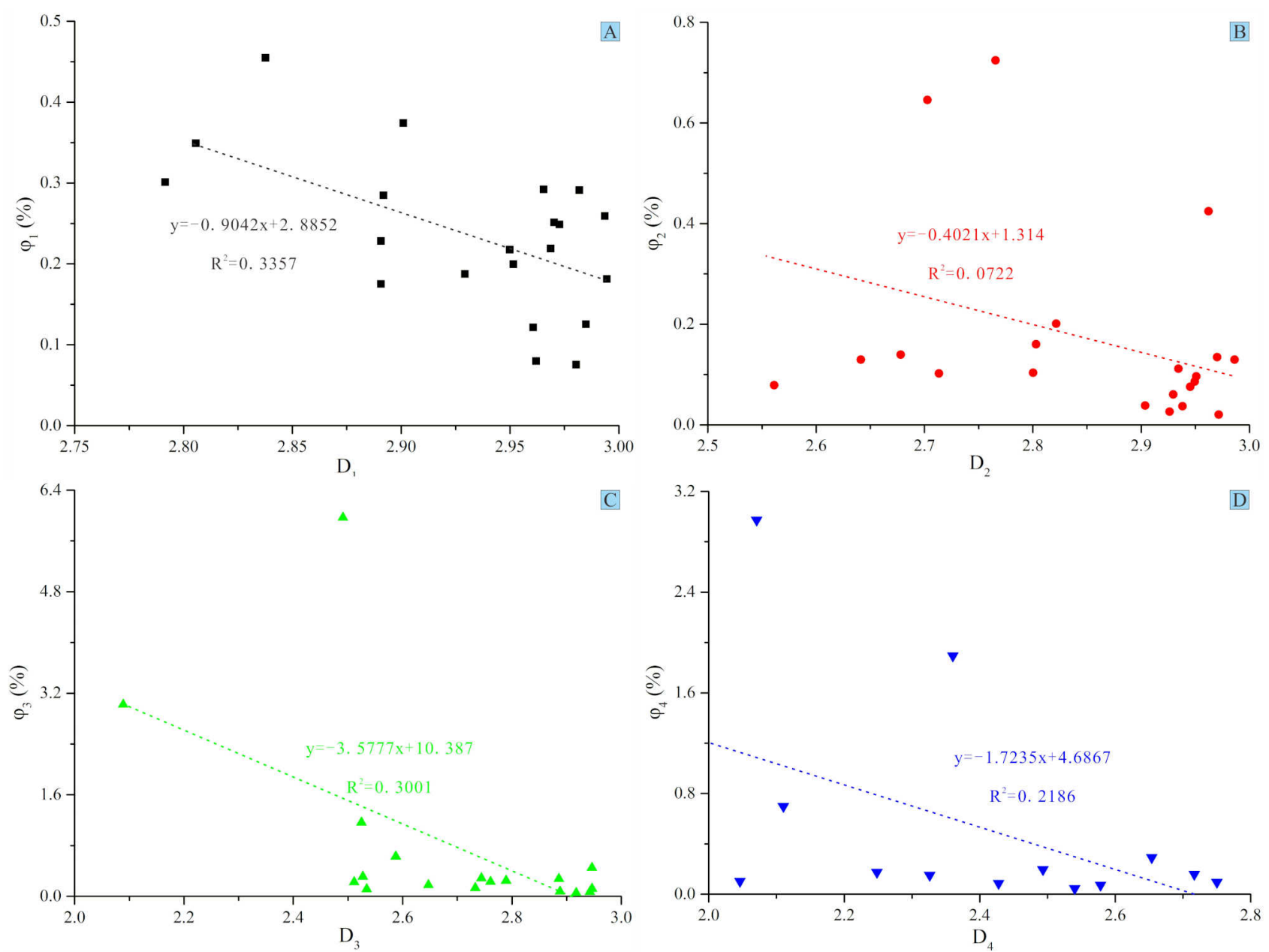

Fig. 15 Relations of $D_{1}(A), D_{2}(B), D_{3}(C)$ and $D_{4}(D)$ with porosity of corresponding pores for carbonate cap rock samples.

$\mathrm{D}_{1}, \mathrm{D}_{3}$, and $\mathrm{D}_{4}$ have good negative correlations with porosity of their corresponding pore sizes ( $\varphi_{1}, \varphi_{3}$ and $\varphi_{4}$ respectively) (Fig. 15A, C, D). On the contrary, $\mathrm{D}_{2}$ has a poor negative correlation with porosity of macropore-II ( $\left.\varphi_{2}\right)$ (Fig. 15B). Fractal dimensions from $\mathrm{D}_{1}$ to $\mathrm{D}_{4}$ show an increasing trend along with decreasing porosity. This suggests that macropores and mesopores make a significant contribution to the fractal variation on the basis of PSD (Schmitt et al., 2013). However, the total fractal dimension ( $\left.\mathrm{D}_{\text {total }}\right)$ shows no apparent relation with porosity (Fig. 16A). This is probably disturbed by transitional pores and micropores together with pore-filling clay mineral (e.g., laminated illite and lamellated illite in Fig. 9C, E). These clays make the pore structure more irregular and weaken the influence of macropores and mesopores on $D_{\text {total }}$ (Aliakbardoust and Rahimpour-Bonab, 2013). In conclusion, different PSDs of carbonate cap rock have varying impacts on their fractal behaviors, whereas only the larger pores have a significantly negative correlation with fractal changes.

The analyses presented here reveal that the different fractal dimensions (from $\mathrm{D}_{1}$ to $D_{6}$ ) and $D_{\text {total }}$ show various characteristics and disparate relationships with their corresponding porosity. The tight carbonate cap rock from the $\mathrm{O}_{1-2} \mathrm{y}$ Formation has a 
Wu et al., 2019. Impact of pore structure and fractal characteristics on the sealing capacity of Ordovician carbonate cap rock in the Tarim Basin, China. Marine and Petroleum Geology, DOI: 10.1016/j.marpetgeo.2019.01.014

pretty complex and heterogeneous pore throat structure with poor connectivity (Krohn, 1988), and the different PSDs of this rock has a strong impact on the fractal features of pore throat.
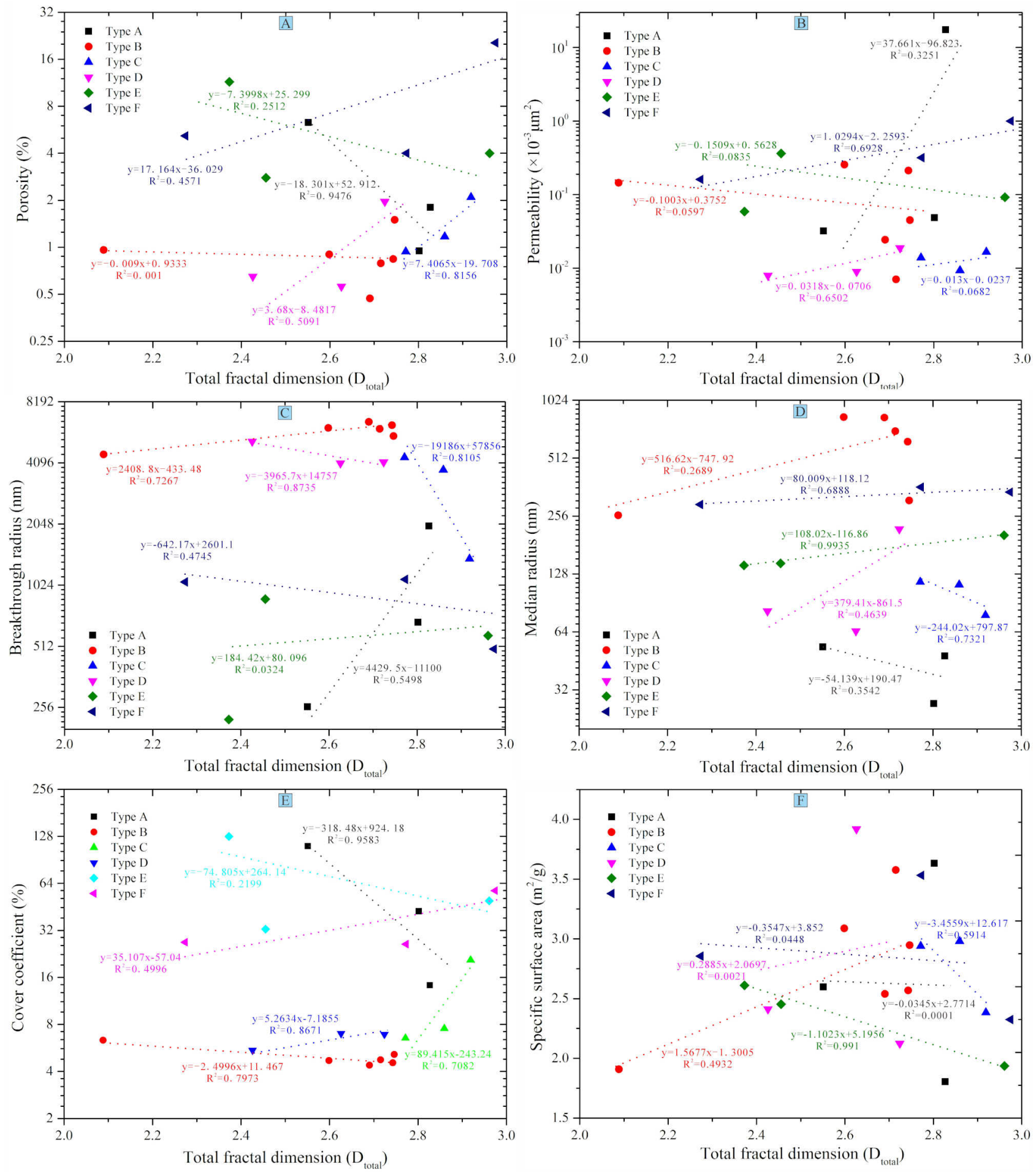

Fig. 16 Correlations of total fractal dimension $\left(\mathrm{D}_{\text {total }}\right)$ with a series of test parameters for 21 Ordovician carbonare cap rock. 
Wu et al., 2019. Impact of pore structure and fractal characteristics on the sealing capacity of Ordovician carbonate cap rock in the Tarim Basin, China. Marine and Petroleum Geology, DOI: 10.1016/j.marpetgeo.2019.01.014

\subsection{Relationship between fractal dimension and sealing capacity}

The relations between test parameters and total fractal dimension in six types of pore throat structures were analyzed to reveal the effect of the total fractal dimension ( $D_{\text {total }}$ ) on the pore throat properties and rock's sealing capacity (Fig. 16). The correlation coefficients of $\mathrm{D}_{\text {total }}$ and testing parameters are listed in Table 3. Porosity presents a good relationship with $\mathrm{D}_{\text {total }}$ in types $\mathrm{A}$ and $\mathrm{C}$ with relatively high correlation coefficients, whereas such correlation is weak for the other four types, especially for type B (Fig. 16A). Permeability has good correlation with $\mathrm{D}_{\text {total }}$ for types $\mathrm{D}$ and F, and a poor one for the other residual types, especially in types B, C and E (Fig. 16B). In general, the porosity and permeability of carbonate, sandstone and shale reservoirs decrease with increasing fractal dimension (Schlueter et al., 1997; He et al., 2016; Li et al., 2017; Zhang et al., 2018). However, we have found here that for tight carbonate cap rocks comprising various pore throat sizes there are significant differences between porosity, permeability and total fractal dimension. This is likely due to the fact that the irregularly distributed pore morphologies make the pore throat complex (Corrado et al., 2014), resulting in a pore system that acts as a baffle for fluid flow due to the transformation from smooth to rough pore throat surfaces (Chalmers and Bustin, 2008; Nooruddin et al., 2014; Norbisrath et al., 2017; Zambrano et al., 2018).

The breakthrough pressure $\left(P_{b}\right)$ has a good relationship with $\mathrm{D}_{\text {total }}$ of types $\mathrm{C}$ and $\mathrm{D}$, but has a poor correlation with that of type E (Fig. 16C). Overall, the median pressure $\left(P_{m}\right)$ and cover coefficient $(\mathrm{CC})$ correlate well with $\mathrm{D}_{\text {total, }}$ especially in types $\mathrm{E}$ and $\mathrm{A}$ that present very high correlation coefficients (Fig. 16D, E). However, the correlation coefficients between specific surface area (SSA) and $\mathrm{D}_{\text {total }}$ vary dramatically (i.e., from 0.0001 to 0.991 ; Fig. 16F). This indicates that the relationships between $P_{b}, P_{m}, \mathrm{CC}$, SSA, and Dtotal are highly complex and difficult to predict. Consequently, as discussed above, cap rocks with similar PSDs and pore radii probably can potentially have completely distinct pore networks (Muller and Mccauley, 1992), thereby showing diverse fractal dimensions (Zhang et al., 2018).

In addition, the plots between various fractal dimensions of corresponding pore throat, and $P_{b}, P_{m}, \mathrm{CC}$ and SSA were analyzed to investigate the variable fractal features and the impact of fractal dimensions on the rock's sealing capacity (Table 4 and Fig. 17, 18, 19). $\mathrm{D}_{1}$ has good relationship with $P_{b}$ and $\mathrm{CC}$ (Fig. 17A1, A2), while $\mathrm{D}_{2}$ and $\mathrm{D}_{3}$ only have good relationship with $P_{m}$ (Fig. 17B1, C1). Furthermore, although $\mathrm{D}_{5}$ only has a good relationship with SSA (Fig. 19F2), D4 has a poor correlation with all these four parameters (Fig. 18D1, D2). The aforementioned analyses reveal that the heterogeneity and anisotropy of macropores are the main controls on the carbonate cap rock sealing capacity. Cap rocks are effective seals for the underlying reservoirs if both are high. Consequently, the increasing $\mathrm{D}_{1}$ not only reflects the roughness degree of pore throat surface and irregularities of pore shapes, but also enhances the capillary 
resistance force to hydrocarbon migration (Vavra et al., 1992; Wu et al., 2018a). The poor relationship of $\mathrm{D}_{\text {total }}$ with these four parameters has resulted from the various positive or negative correlations of five fractal dimensions (from $\mathrm{D}_{1}$ to $\mathrm{D}_{5}$ ) with these parameters.
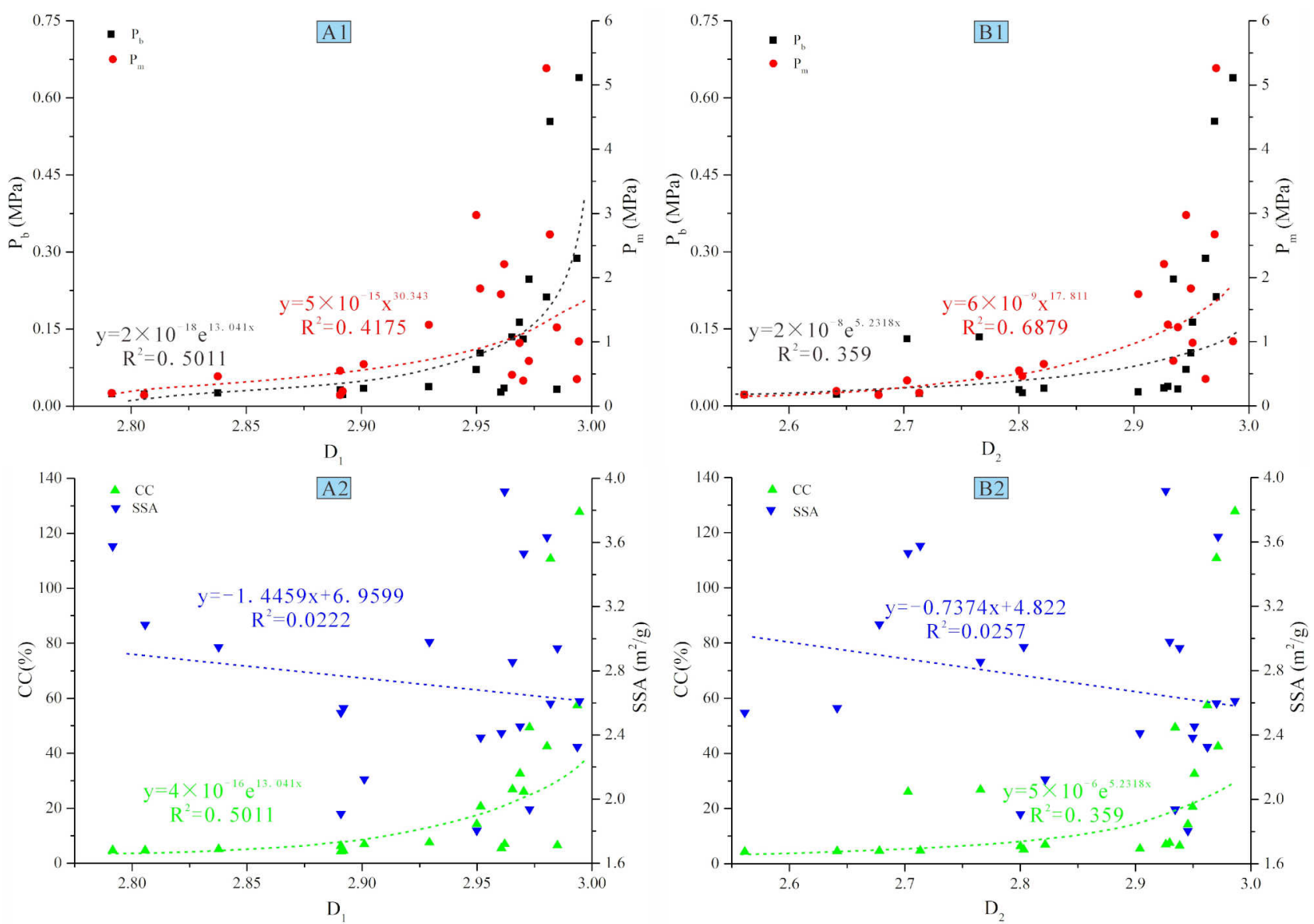

Fig. 17 Correlations of $\mathrm{D}_{1}$, and $\mathrm{D}_{2}$ with $P_{b}, P_{m}, \mathrm{CC}$ and SSA of Ordovician carbonate cap rock samples from the Tahe oilfield.

The pore throat structure of carbonate cap rock has fractal characteristics in a certain scale range as a consequence of the residual bitumen (Fig. 2L), pore-filling and/or pore-bridging illites (Fig. 9C, E) (Wang et al., 2015; Java et al., 2005). Calcite cements and illite occupying some part of the pore space make pores appear with an irregular shape, thus increasing the fractal dimension (Zhang et al., 2018). Our results reveal that the larger the fractal dimension of pore properties is, the rougher the pore surface is, and the more irregular the PSD is (Norbisrath et al., 2017). We have also found that the more irregular the pore throat shape is, the greater the resistance for hydrocarbon fluid flow is (Schmitt et al., 2013). Fig. 20 shows that the fractal dimensions corresponding to the macropores in the type B pore structure increase with the increasing of macroporosity of cap rock, which implies the cap rock heterogeneity become stronger for the Ordovician Yingshan Formation in the Tarim Basin. In addition, the macroporosity within the carbonate cap rock presents an exponential correlation with the height of gas column (HGC) with a higher correlation coefficient. This 
suggests that the increase of sealing capacity occur in the type B pore structure of carbonate cap rock as a consequence of increasing heterogeneity (Fig. 20). Consequently, the range variation of porosity and permeability can be determined for the increase in macroporosity with larger pore throat diameters enhancing the cap rock heterogeneity: the porosity ranges from $0.25 \%$ to $0.85 \%$ and the permeability ranges from $0.004 \times 10^{-3} \mu^{2}$ to $0.138 \times 10^{-3} \mu \mathrm{m}^{2}$. Strong anisotropy and poor connectivity of pore throat structures in carbonates is key for the development of an effective seal (Zhou et al., 2017). The enhancement of carbonate cap rock heterogeneity can potentially improve the resistance of the rock to hydrocarbon migration (Wu et al., 2018a), thus better sealing oil and gas in the underlying fracture-cavity carbonate reservoirs (Schlömer and Krooss, 1997).
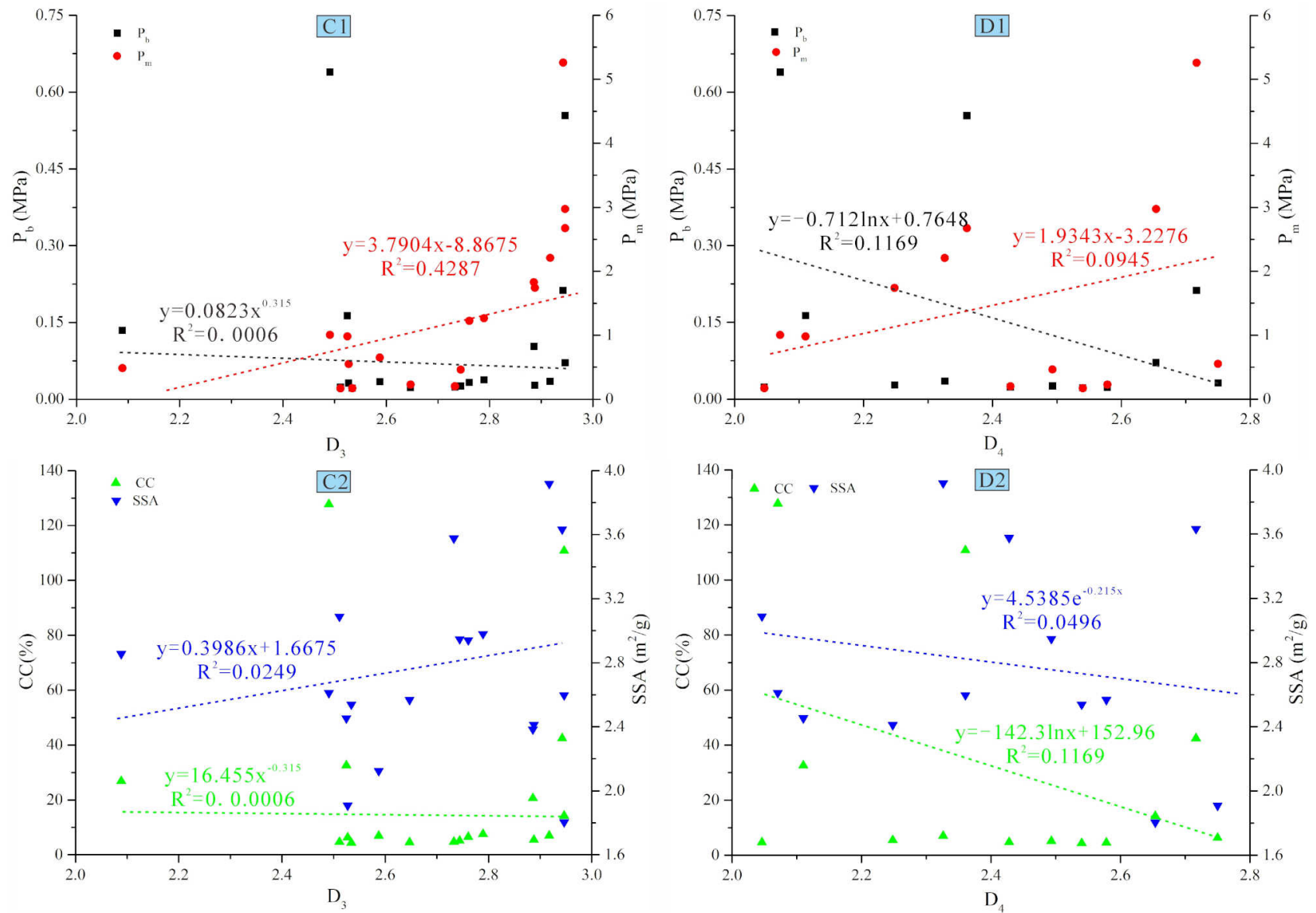

Fig. 18 Correlations of $\mathrm{D}_{3}$ and $\mathrm{D}_{4}$ with $P_{b}, P_{m}, \mathrm{CC}$ and SSA of Ordovician carbonate cap rock samples from the Tahe oilfield. 
Wu et al., 2019. Impact of pore structure and fractal characteristics on the sealing capacity of Ordovician carbonate cap rock in the Tarim Basin, China. Marine and Petroleum Geology, DOI: 10.1016/j.marpetgeo.2019.01.014
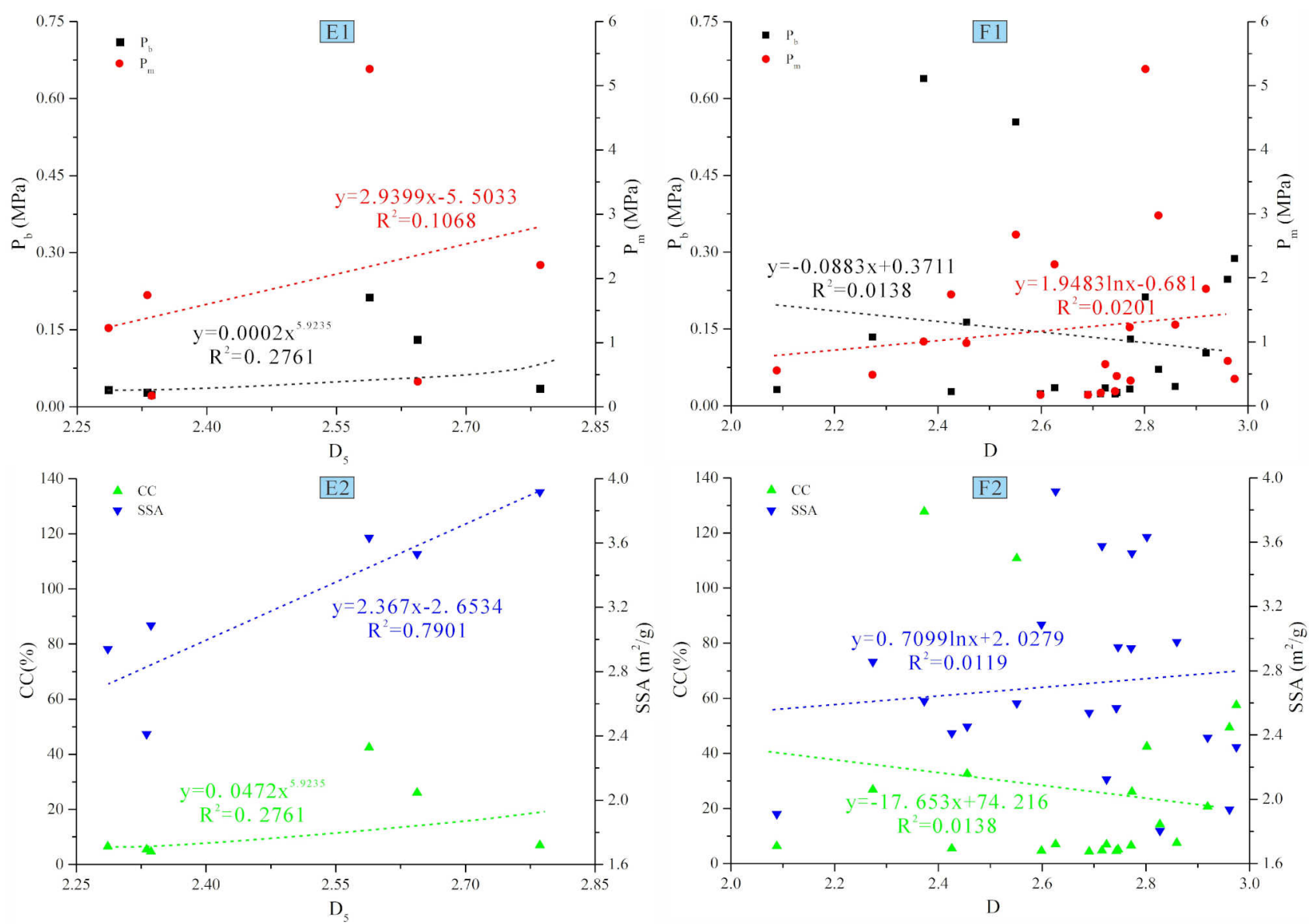

Fig. 19 Correlations of $\mathrm{D}_{5}$ and $\mathrm{D}_{\text {total }}$ with $P_{b}, P_{m}, \mathrm{CC}$ and SSA of Ordovician carbonate cap rock samples from the Tahe oilfield.

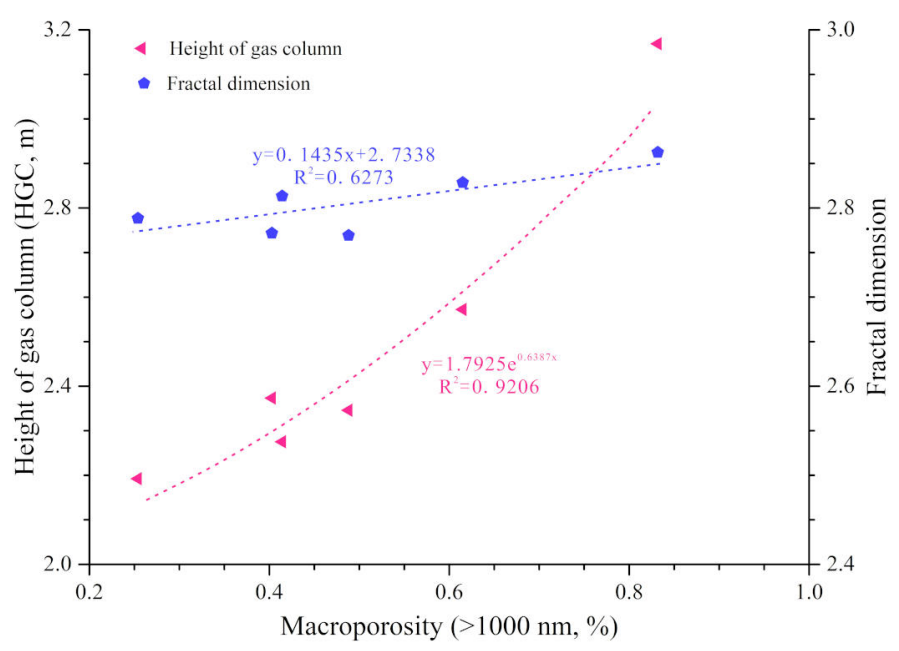

Fig. 20 Relationships of macroporosity $(>1,000 \mathrm{~nm})$, height of gas column (HGC), and fractal dimension corresponding to macropore-I $(>2,500 \mathrm{~nm})$ and macropore-II $(>1,000 \mathrm{~nm})$ in the type B pore structure of carbonate cap rock from the Tarim Basin. 


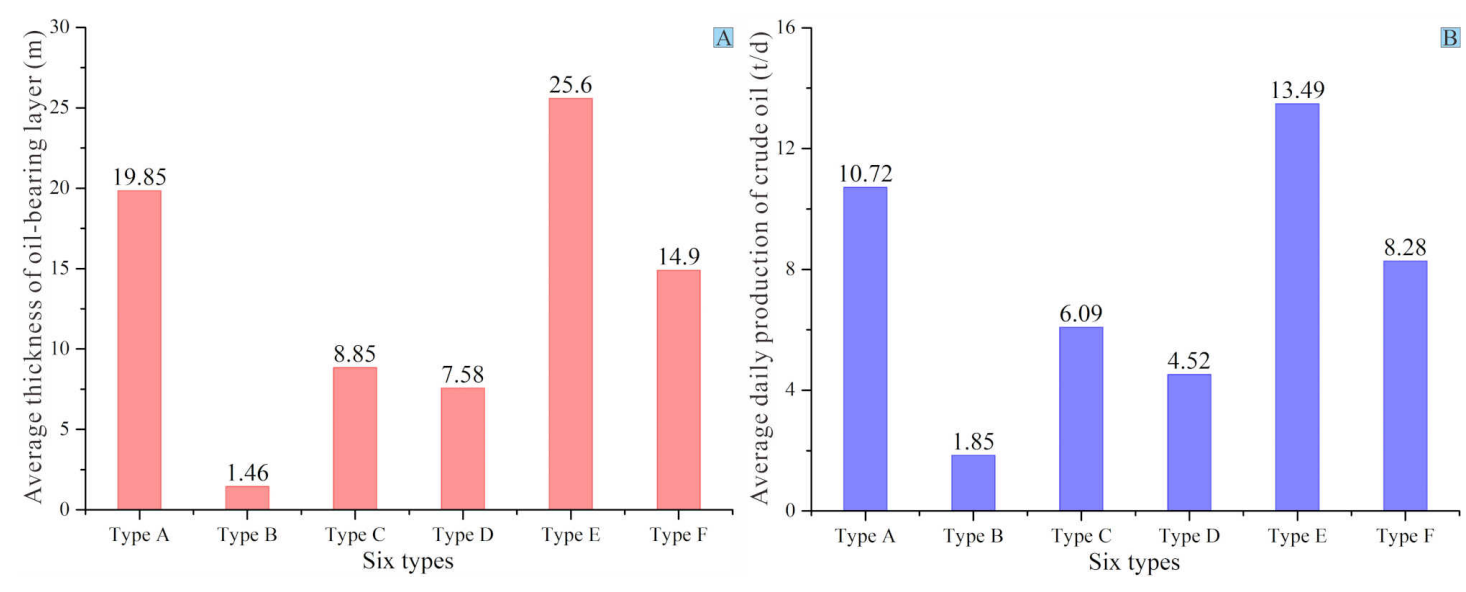

Fig. 21 Average thickness of the oil-bearing layer (A) and average daily production of crude oil (B) sealed by the carbonate cap rock intervals containing six types of pore throat structures defined from the Tahe oilfield in the Tarim Basin.

\subsection{Relationship between sealing capacity and actual production}

Evaluating the influence of various types of pore structures and their fractal dimension on the height of gas column (HGC) in actual production is necessary to analyze the sealing capacity of carbonate cap rock, and then to predict the favorable distribution zones of cap rock that seal the underlying hydrocarbon in carbonate reservoirs. Consequently, we have collected some actual production data from the Tahe oilfield in the Tarim basin. These data are primarily the average thickness of the oilbearing layers that are sealed by these cap rock intervals where the studied samples were collected from. We have also analyzed the average daily production of crude oil from the oil-bearing layers in carbonate reservoirs.

There is a positive correlation between the sealing capacity and the average thickness of the oil-bearing layer. The cap rock interval with pore throat structure type E containing the maximum calculated HGC (Fig. 3) can seal the underlying oil-bearing layer with the largest average thickness of $25.6 \mathrm{~m}$ (Fig. 21A). Importantly, this oilbearing interval has the largest average daily yield of crude oil with 13.49 ton per day (Fig. 21B). However, the cap rock interval corresponding to the minimum calculated HGC of pore throat structure B merely covers the thinnest average oil-bearing interval of $1.46 \mathrm{~m}$, and the average daily output of crude oil is only $1.85 \mathrm{t} / \mathrm{d}$ (Fig. 21B). This implies that the presence of a complex pore throat structure has a significant influence on the vertical distribution of oil-bearing layers and on actual production. The difference in the pore structure of the overlying carbonate cap rock causes a large change of the sealing performance. This does not only results in a change of the thickness of the oil-bearing layers but also affects the daily output of oil and gas in carbonate reservoirs. 
Carbonate cap rocks acting as local cap rocks, which are different from traditional cap rocks (e.g. mudstone cap rocks and evaporite cap rocks) and are controlled by depositional settings, diagenesis, lithological types, tectonic movements and fractures (Wu et al., 2018b). Numerous explorations have revealed the sealing potential of carbonate cap rocks in the Tahe oilfield from the Tarim Basin (Wu et al., 2018a). A complete reference to the present traditional evaluation system and evaluation parameters of cap rock for the medium and large gas fields in China (Lü et al., 2005; Hu et al., 2009; Qian et al., 2012) is not applicable to the sealing capacity assessment of carbonate cap rock. Hence, new evaluation criteria of sealing capacity for carbonate cap rock should be established in the following study.

In summary, the fractal theory is used here to study the complexity of pore systems with irregular shape. The fractal dimension can not only describe the pore connectivity and complexity of pore throat structures, but also describes the particle size composition of the reservoir rocks. Therefore, the fractal dimension becomes an important parameter to quantitatively investigate the microscopic pore regular structure and combination characteristics, as well as to characterize the microscopic heterogeneity and sealing capacity of carbonate cap rock.

\section{Conclusions}

(1) The carbonate cap rock of the $\mathrm{O}_{1-2} \mathrm{y}$ Formation in the Tahe oilfield is mainly dominated by micrite, followed by calcarenite, dolomitic limestone and silty-fine crystalline dolomite. The pore space of the carbonate cap rock is mainly in the form of macropores $(>1,000 \mathrm{~nm})$, mesopores $(100-1,000 \mathrm{~nm})$ and transitional pores (10-100 $\mathrm{nm}$ ), and can be classified in six types from type A to F with each having a distinct pore size distribution (PSD). A wide range of PSD characteristics and an extremely weak correlation between porosity and permeability suggests that the pore throat structures of this carbonate cap rock are rather complex and poorly interconnected.

(2) The fractal theory is suitable for the characterization of pore throat structures of carbonate cap rocks. The fractal characteristics of the studied cap rock can be divided into six segments, which correspond to the fractal dimensions $\mathrm{D}_{1}, \mathrm{D}_{2}, \mathrm{D}_{3}, \mathrm{D}_{4}, \mathrm{D}_{5}$, and $\mathrm{D}_{6}$, with discrete PSDs. Overall, the decreasing average fractal dimension values from $\mathrm{D}_{1}$ to $\mathrm{D}_{6}$ are associated with a decrease in pore throat size. $\mathrm{D}_{\text {total }}$ in the range of 2.08852.9609 (with an average of 2.6618) shows that the pore networks of carbonate cap rock are strongly heterogeneous and significantly anisotropic.

(3) The average fractal dimensions of micrite and silty-fine crystalline dolomite are significantly lower than those of calcarenite and dolomitic limestone, and thus the pore throat structures of this carbonate cap rock are affected by lithological types. The sealing height of gas column for carbonate cap rock are reduced with the increasing of 
microfracture density. Abundant micorfractures possess an adverse impact on the sealing performance of this rock. Conversely, the height of gas column is positively correlated with the cement content. The good sealing capacity of the cap rock in the studied area resulted from the high cement content.

(4) Well-developed mesopore-II, transitional pore-I and transitional pore-II have a significant control on the breakthrough pressure and affect the trigger threshold of hydrocarbon migration within carbonate reservoirs. Mesopores play a more significant role in preventing petroleum transport than macropores do. Comparisons between $\mathrm{D}_{1}$, $\mathrm{D}_{2}, \mathrm{D}_{3}, \mathrm{D}_{4}, \mathrm{D}_{5}$, and $\mathrm{D}_{6}$ suggest that meso-pore and transitional pore structure types with fractal dimensions ( $\mathrm{D}_{3}$ and $\mathrm{D}_{4}$ ) close to 2.0 are more homogeneous and less complex than macropore structures with those $\left(\mathrm{D}_{1}\right.$ and $\left.\mathrm{D}_{2}\right)$ close to 3.0. $\mathrm{D}_{1}, \mathrm{D}_{3}$ and $\mathrm{D}_{4}$ show a better negative trend with porosity decreasing than $\mathrm{D}_{2}$.

(5) Different pore size distributions of carbonate cap rock have varying impacts on their fractal behavior, whereas only the larger pores (macropores and mesopores) have a significant negative correlation with fractal changes. The total fractal dimension ( $D_{\text {total }}$ shows no apparent relation with porosity, which is disturbed by transitional pores and micropores together with pore-filling clay mineral that make the pore structure more irregular and weaken the influence of macropores and mesopores on $\mathrm{D}_{\text {total }}$.

(6) The carbonate cap rock comprising various PSDs has significant differences between porosity, permeability and total fractal dimension, meanwhile the relationships between breakthrough pressure, median pressure, cover coefficient, specific surface area and $\mathrm{D}_{\text {total }}$ are highly complex. We conclude that the sealing capacity of cap rock is mainly controlled by the heterogeneity and anisotropy of macropores.

\section{Acknowledgements}

This study was supported by the Major National Science-Technology Projects of China (Nos. 2017ZX05005-002-003 and 2017ZX05009-002), China Scholarship Council (No. 201806400037), National Natural Science Foundation of China (No. 51574208), Strategic Priority Research Program of the Chinese Academy of Sciences (No. XDA14010201-02) and Fundamental Research Funds for the Central Universities (No. 2-9-2015-141). Many thanks to Xinxin Yin and Xue Fan for their help in rock samples preparation. We are grateful to the Editor-in-Chief Qinhong $\mathrm{Hu}$, Associate Editor Hugh Daigle and three anonymous reviewers for their constructive suggestions and critical comments that significantly improved the manuscript. 
Wu et al., 2019. Impact of pore structure and fractal characteristics on the sealing capacity of Ordovician carbonate cap rock in the Tarim Basin, China. Marine and Petroleum Geology, DOI: 10.1016/j.marpetgeo.2019.01.014

\section{References}

Aliakbardoust, E., Rahimpour-Bonab, H., 2013. Effects of pore geometry and rock properties on water saturation of a carbonate reservoir. Journal of Petroleum Science and Engineering. 112(3), 296-309.

Anselmetti, F.S., Luthi, S., Eberli, G.P., 1998. Quantitative characterization of carbonate pore systems by digital image analysis. AAPG Bulletin. 82(10), 1815-1836.

Armitage, P.J., Worden, R.H., Faulkner, D.R., Aplin, A.C., Butcher, A.R., Iliffe, J., 2010. Diagenetic and sedimentary controls on porosity in Lower Carboniferous fine-grained lithologies, Krechba field, Algeria: A petrological study of a caprock to a carbon capture site. Marine and Petroleum Geology. 27(7), 1395-1410.

Berg, R.R., 1975. Capillary pressures in stratigraphic traps. AAPG Bulletin. 59(6), 939-956.

Boulin, P.F., Bretonnier, P., Vassil, V., Samouillet, A., Fleury, M., Lombard, J.M., 2013. Sealing efficiency of caprocks: Experimental investigation of entry pressure measurement methods. Marine and Petroleum Geology. 48: 20-30.

Budd, D.A., 2002. The relative roles of compaction and early cementation in the destruction of permeability in carbonate grainstones: A case study from the Paleogene of West-Central Florida, U.S.A. Journal of Sedimentary Research. 72 (1), 116-128.

Caillet, G., 1993. The caprock of the Snorre Field, Norway: a possible leakage by hydraulic fracturing. Marine and Petroleum Geology. 10(1), 42-50.

Cao, T.T., Song, Z.G., Wang, S.B., Cao, X.X., Li, Y., Xia, J., 2015. Characterizing the pore structure in the Silurian and Permian shales of the Sichuan Basin, China. Marine and Petroleum Geology, 61: 140-150.

Cao, T.T., Song, Z.G., Liu, G.X., Yin, Q., Luo, H.Y., 2016. Characteristics of shale pores, fractal dimension and their controlling factors determined by nitrogen adsorption and mercury injection methods. Petroleum Geology and Recovery Efficiency. 23(2), 1-8.

Colón, C.F.J., Oelkers, E.H., Schott, J., 2004. Experimental investigation of the effect of dissolution on sandstone permeability, porosity, and reactive surface area. Geochimica et Cosmochimica Acta. 68(4), 805-817.

Corrado, S., Aldega, L., Celano, A.S., Benedetti, A.A.D., Giordano, G., 2014. Cap rock efficiency and fluid circulation of natural hydrothermal systems by means of XRD on clay minerals (Sutri, Northern Latium, Italy). Geothermics. 50: 180-188.

Chalmers, G.R.L., Bustin, R.M., 2008. Lower Cretaceous gas shales in northeastern British Columbia, Part I: geological controls on methane sorption capacity. Bulletin of Canadian Petroleum Geology. 56(1), 1-21.

Cheng, Q.Q., Chen, H.Y., Fan M., Wang, Q., Chen, W.J., 2006. Determination of the total pore texture of caprock. Petroleum Geology and Experiment. 28(6), 604-608.

Fan, M., Chen, H.Y., Yu, L.J., Zhang, W.T., Liu, W.X., Bao, Y.J., 2011. Evaluation standard of mudstone caprock combining specific surface area and breakthrough pressure. Petroleum Geology and Experiment. 33 (1), 87-90.

Dutta, T., Tarafdar, S., 2003. Fractal pore structure of sedimentary rocks: Simulation by ballistic deposition. Journal of Geophysical Research. 108(B2), 1-6. 
Wu et al., 2019. Impact of pore structure and fractal characteristics on the sealing capacity of Ordovician carbonate cap rock in the Tarim Basin, China. Marine and Petroleum Geology, DOI: 10.1016/j.marpetgeo.2019.01.014

Ehrenberg, S.N., Nadeau, P.H., 2005. Sandstone vs. carbonate petroleum reservoirs: a global perspective on porosity-depth and porosity-permeability relationships. AAPG Bulletin. 89(4), 435-445.

Erwin, C.R., Eby, D.E., Whitesides, V. S., 1979. Clasticity index: A key to Correlating Depositional and Diagenetic Environments of Smackover Reservoirs, Oaks Field, Claiborne Parish, Louisiana. Gulf Coast Association of Geological Societies Transactions, Vol. 29, pp. 52-62.

Esteban, M., Taberner, C., 2003. Secondary porosity development during late burial in carbonate reservoirs as a result of mixing and/or cooling of brines. Journal of Geochemical Exploration, 78(3), 355-359.

Gundogar, A.S., Ross, C.M., Akin, S., Kovscek, A.R., 2016. Multiscale pore structure characterization of middle east carbonates. Journal of Petroleum Science and Engineering. 146, 570-583.

He, J.H., Ding, W.L., Li, A., Sun, Y.X., Dai, P., Yin, S., Chen, E., Gu, Y., 2016. Quantitative microporosity evaluation using mercury injection and digital image analysis in tight carbonate rocks: A case study from the Ordovician in the Tazhong Palaeouplift, Tarim Basin, NW China. Journal of Natural Gas Science \& Engineering. 34, 627-644.

Hu, G.Y., Wang, X.B., Wang, Y.F., Tao, X.W., Ni, Y.Y., Yang, C., 2009. Cap rock characteristics of medium and large gas fields in China. Natural Gas Geoscience. 20(2), 162-166.

Huq, F., Smalley, P.C., Mørkved, P.T., Johansen, I., Yarushina, V., Johansen, H., 2017. The Longyearbyen $\mathrm{CO}_{2} \mathrm{Lab}$ : Fluid communication in reservoir and caprock. International Journal of Greenhouse Gas Control. 63, 59-76.

Jaya, I., Sudaryanto, A., Widarsono, B., 2005. Permeability prediction using pore throat and rock fabric: A Model from Indonesian reservoirs. SPE Asia Pacific Oil and Gas Conference and Exhibition.

Javanbakht, M., Wanas, H.A., Jafarian, A., Shahsavan, N., Sahraeyan, M., 2018. Carbonate diagenesis in the Barremian-Aptian Tirgan Formation (Kopet-Dagh Basin, NE Iran): Petrographic, geochemical and reservoir quality constraints. Journal of African Earth Sciences. $144,122-135$.

Kang, Y.Z., 2005. Cases of Discovery and Exploration of Marine Fields in China (Part 4): Tahe Oilfield in Tarim Basin. Marine Origin Petoleum Geology. 10(4), 31-38.

Krohn, C.E., 1988. Fractal measurements of sandstones, shales, and carbonates. Journal of Geophysical Research Solid Earth. 93(B4), 3297-3305.

Li, P., Zheng, M., Bi, H., Wu, S.T., Wang, X.R., 2017. Pore throat structure and fractal characteristics of tight oil sandstone: A case study in the Ordos Basin, China. Journal of Petroleum Science and Engineering. 149: 665-674.

Li, S., Dong, M., Li, Z., Huang, S., Qing, H., Nickel, E., 2005. Gas breakthrough pressure for hydrocarbon reservoir seal rocks: implications for the security of long-term $\mathrm{CO}_{2}$ storage in the weyburn field. Geofluids. 5(4), 326-334.

Liu, X.P., Jin, Z.J., Bai, G.P., Guan, M., Liu, J., Pan, Q.H., Li, T., Xing, Y.J., 2017. Formation and distribution characteristics of Proterozoic-Lower Paleozoic marine giant oil and gas fields worldwide. Petroleum Science. 14(2): 237-260.

Loucks, R.G., Reed, R.M., Ruppel, S.C., Hammes, U., 2012. Spectrum of pore types and networks 
Wu et al., 2019. Impact of pore structure and fractal characteristics on the sealing capacity of Ordovician carbonate cap rock in the Tarim Basin, China. Marine and Petroleum Geology, DOI: 10.1016/j.marpetgeo.2019.01.014

in mudrocks and a descriptive classification for matrix-related mudrock pores. AAPG Bulletin. 96(6), 1071-1098.

Lü, X.X., Wang, Y.F., Yu, H.F., Bai, Z.K., 2017. Major factors affecting the closure of marine carbonate caprock and their quantitative evaluation: A case study of Ordovician rocks on the northern slope of the Tazhong uplift in the Tarim Basin, western China. Marine and Petroleum Geology. 83, 231-245.

Lü, Y.F., Fu, G., Yu, D., 2005. Comprehensive evaluation of sealing ability of cap rock in China's large and medium gas fields and their contribution to gas accumulation. Oil \& Gas Geology. 26(6), 742-741.

Mandelbrot, B.B., Passoja, D.E., Paullay, A.J., 1984. Fractal character of fracture surfaces of metals. Nature, 308, 721-722.

Mazzullo, S.J., Harris, P.M., 1992. Mesogenetic dissolution: its role in porosity development in carbonate reservoirs. AAPG Bulletin. 76(5), 607-620.

Muller, J., Mccauley, J.L., 1992. Implication of fractal geometry for fluid flow properties of sedimentary rocks. Transport in Porous Media. 8(2), 133-147.

Newell, P., Martinez, M.J., Eichhubl, P., 2017. Impact of layer thickness and well orientation on caprock integrity for geologic carbon storage. Journal of Petroleum Science and Engineering. $155,100-108$.

Nooruddin, H.A., Hossain, M.E., Al-Yousef, H., Okasha, T., 2014. Comparison of permeability models using mercury injection capillary pressure data on carbonate rock samples. Journal of Petroleum Science and Engineering. 121, 9-22.

Norbisrath, J.H., Weger, R.J., Eberli, G.P., 2017. Complex resistivity spectra and pore geometry for predictions of reservoir properties in carbonate rocks. Journal of Petroleum Science and Engineering. 151, 455-467.

Pant, L.M., Huang, H., Secanell, M., Larter, S., Mitra, S.K., 2015. Multi scale characterization of coal structure for mass transport. Fuel. 159, 315-323.

Qian, Y.Y., He, Z.L., Chen, Q.L., Li, H.L., Lu, Q.H., Cai, X.Y., You, D.H., 2012. Sealing capacity of the Ordovician carbonate rocks in Tazhong area, the Tarim Basin. Oil \& Gas Geology. 33(1), $1-9+18$

Rashid, F., Glover, P.W.J., Lorinczi, P., Hussein, D., Lawrence, J.A., 2017. Microstructural controls on reservoir quality in tight oil carbonate reservoir rocks. Journal of Petroleum Science and Engineering. 156, 814-826.

Rezaeyan, A., Tabatabaei-Nejad, S.A., Khodapanah, E., Kamari, M., 2015. A laboratory study on capillary sealing efficiency of iranian shale andanhydrite caprocks. Marine and Petroleum Geology. 66, 817-828.

Ross, D.J. K., Bustin, R.M., 2009. The importance of shale composition and pore structure upon gas storage potential of shale gas reservoirs. Marine and Petroleum Geology. 26(6), 916-927.

Rouquerol, J., Avnir, D., Fairbridge, C.W., Everett, D.H., Haynes, J.H., Pernicone, N., Ramsay, J.D.F., Sing, K.S.W., Unger, K.K., 1994. Recommendations for the characterization of porous solids (Technical Report). Pure and Apply Chemistry. 66(8), 1739-58.

Schlömer, S., Krooss, B.M., 1997. Experimental characterisation of the hydrocarbon sealing efficiency of cap rocks. Marine and Petroleum Geology. 14(5), 565-580. 
Wu et al., 2019. Impact of pore structure and fractal characteristics on the sealing capacity of Ordovician carbonate cap rock in the Tarim Basin, China. Marine and Petroleum Geology, DOI: 10.1016/j.marpetgeo.2019.01.014

Schlueter, E.M., Zimmerman, R.W., Witherspoon, P.A., Cook, N.G.W., 1997. The fractal dimension of pores in sedimentary rocks and its influence on permeability. Engineering Geology. 48(3), 199-215.

Schmitt, M., Fernandes, C.P., Neto, J.A.B.D.C., Wolf, F.G., Santos, V.S.S.D., 2013. Characterization of pore systems in seal rocks using nitrogen gas adsorption combined with mercury injection capillary pressure techniques. Marine and Petroleum Geology. 39(1), 138-149.

Sing, K.S.W., Everett, D.H., Haul, R.A.W., Moscou, L., Pierotti, R.A., Rouquerol, J., Siemieniewska, T., 1985. Reporting physisorption data for gas/solid systems-with special reference to the determination of surface area and porosity. Pure \& Applied Chemistry. 57(4), 603-619.

Thompson, A.H., Katz, A.J., Krohn. C.E., 1987. The microgeometry and transport properties of sedimentary rock. Advances in Physics. 36(5), 625-694.

Tian, F., Jin, Q., Lu, X.B., Lei, Y.H., Zhang, L.K., Zheng, S.Q, Zhang, H.F., Rong, Y.S., Liu, N.G., 2016. Multi-layered ordovician paleokarst reservoir detection and spatial delineation: A case study in the Tahe Oilfield, Tarim Basin, Western China. Marine and Petroleum Geology. 69, $53-73$

Vavra, C.L., Kaldi, J.G., Sneider, R.M., 1992. Geological applications of capillary pressure: a review. AAPG Bulletin. 76:6(6), 840-850.

Wang, G.W., Li, P.P., Hao, F., Zou, H.Y., Zhang, L., Yu, X.Y., 2015. Impact of sedimentology, diagenesis, and solid bitumen on the development of a tight gas grainstone reservoir in the Feixianguan Formation, Jiannan area, China: Implications for gas exploration in tight carbonate reservoirs. Marine and Petroleum Geology, 64: 250-265.

Wu, J., Fan, T.L., Gao, Z.Q., Gu, Y., Wang, J.B., Du, Y., Li, C., Wang, S.S., Zhang, C.J., Meng, M.M., Wei, D., Fan, H., 2018a. Identification and characteristic analysis of carbonate cap rock: A case study from the Lower-Middle Ordovician Yingshan Formation in Tahe oilfield, Tarim Basin, China. Journal of Petroleum Science and Engineering. 164: 362-381.

Wu, J., Fan, T.L., Gao, Z.Q., Yin, X.X., Fan, X., Li, C.C., Yu, W.Y., Li, C., Zhang, C.J., Zhang, J.H., Sun, X.N., 2018b. A conceptual model to investigate the impact of diagenesis and residual bitumen on the characteristics of Ordovician carbonate cap rock from Tarim Basin, China. Journal of Petroleum Science and Engineering.168: 226-245.

Xie, S.Y., He, Z.L., Qian, Y.X., Fang, H.P., Zhang, T.F., Zhang, D.W., Wo, Y.J., Bao, Z.Y., 2015. Multifractality of 3D pore structures of carbonate rocks based on CT images. Journal of Geology. 39 (1), 46-54.

Xoaoth, B.B., 1966. Coal and gas outburst. In: Song, S., Wang, Y. (Eds.), Translation, Beijing, China Industry Press.

Yang, C., Zhang, J.C., Wang, X.Z., Tang, X., Chen, Y.C., Jiang, L.L., Gong, X., 2017. Nanoscale pore structure and fractal characteristics of a marine-continental transitional shale: A case study from the lower Permian Shanxi Shale in the southeastern Ordos Basin, China. Marine and Petroleum Geology. 88: 54-68.

Zambrano, M., Tondi, E., Mancini, L., Arzilli, F., Lanzafame, G., Materazzi, M., Torrieri, S., 2017. 3D pore-network quantitative analysis in deformed carbonate grainstones. Marine and Petroleum Geology. 82, 251-264. 
Wu et al., 2019. Impact of pore structure and fractal characteristics on the sealing capacity of Ordovician carbonate cap rock in the Tarim Basin, China. Marine and Petroleum Geology, DOI: 10.1016/j.marpetgeo.2019.01.014

Zhang, J.Z., Li, X.Q., Wei, Q., Gao, W.J., Liang, W.L., Wang, Z., Wang, F.Y., 2017. Quantitative characterization of pore-fracture system of organic-rich marine-continental shale reservoirs: A case study of the Upper Permian Longtan Formation, Southern Sichuan Basin, China. Fuel. 200, 272-281.

Zhang, K., Pang, X.Q., Zhao, Z.F., Shao, X.H., Zhang, X., Li, W., Wang, K., 2018. Pore structure and fractal analysis of Lower Carboniferous carbonate reservoirs in the Marsel area, ChuSarysu basin. Marine and Petroleum Geology. 93, 451-467.

Zhou, X.H., Ding, W.L., He, J.H., Li, A., Sun, Y.X., Wang, X.H., 2017. Microfractures in the middle Carboniferous carbonate rocks and their control on reservoir quality in the Zanaral Oilfield. Marine and Petroleum Geology. 92: 462-476. 